\title{
Linear hydrodynamic modelling of arrays of submerged oscillating cylinders
}

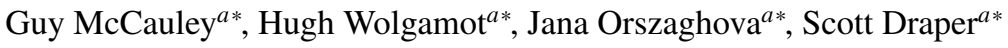 \\ ${ }^{a}$ Oceans Graduate School, The University of Western Australia, Perth, Australia
}

\begin{abstract}
The radiation and diffraction problems for an array of submerged circular cylinders with vertical axes of revolution are formulated exactly to first order in the frequency domain. Matched eigenfunction expansions and a transform matrix method are used to solve the scattering problem for an arbitrary array using a truncated system of matrix equations. For a single shallowly submerged cylinder, the model is used to characterise the occurrence of resonances in the region of fluid above the cylinder moving in heave and surge motion. The method is then applied to a square array of four cylinders, and the effect of array interactions demonstrated. It is found that fluid resonances above the cylinder are still important, but are modified by multiple scattering. Finally, the mean vertical drift force is calculated from the first order solution by direct pressure integration over the body surface.
\end{abstract}

Keywords:

Submerged cylinders, Water waves, Trapped modes, Wave energy, Arrays, Mean vertical drift force

\section{Introduction}

Some wave energy devices and offshore structures can be modelled as submerged oscillating circular cylinders with vertical axes of revolution. This paper presents a semi-analytical method to model an array of such structures using linear theory, in which the multiple body scattering problem is solved by the use of a transform matrix and a system of matrix equations. The use of such a transform matrix method requires only that the diffraction and radiation problem be formulated exactly for a single cylinder; the complete array solution can then be solved simultaneously and exactly. This type of model can be used to analyse the hydrodynamics of large arrays and offers some advantages over traditional boundary element methods when changing the cylinder or array geometry as re-meshing is not required.

This work is partly motivated by the wave energy device under development by Carnegie Clean Energy, the CETO wave energy converter. This device consists of a shallowly submerged cylinder tethered to the sea floor, see figure 1 . The CETO device may be installed in arrays with relatively small spacing; we must therefore consider the effects of device interactions when modelling the hydrodynamics of such an array as this may be important for design of the system for optimum power production (e.g. Borgarino et al. [1]).

\footnotetext{
${ }^{*}$ Corresponding author

Email address: guy.mccauley@research.uwa.edu.au (Guy McCauley ${ }^{a}$ )
} 


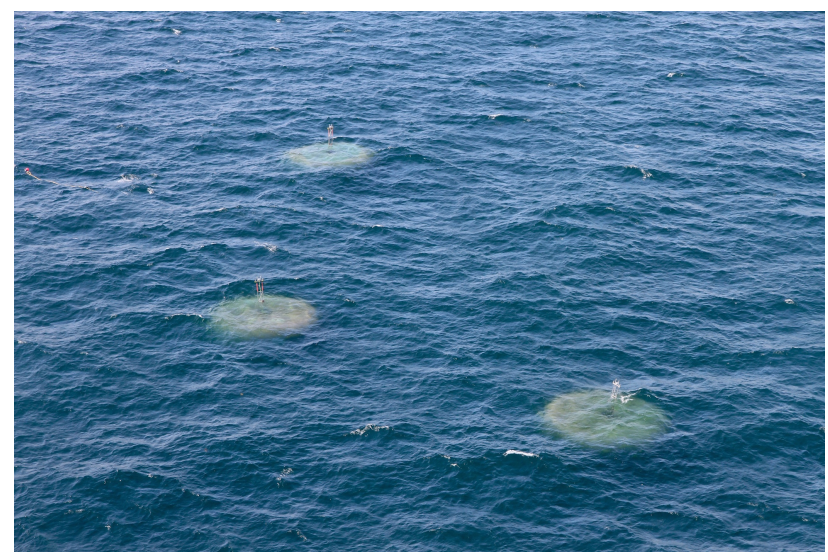

Figure 1: Carnegie Clean Energy CETO wave energy devices. From Carnegie Clean Energy [2].

A submerged oscillating cylinder has significantly altered hydrodynamic properties compared to the same structure floating at the surface due to the layer of fluid above the cylinder. The phenomenon of negative added mass can be observed for very shallow submergences and resonances in the region of fluid above the cylinder can produce large peaks in the damping, as observed by Newman et al. [3] and McIver and Evans [4] for the 2-dimensional and 3-dimensional radiation problems respectively and Martin and Farina [5] for an oscillating submerged plate.

Linear modelling of diffraction around cylinders under the influence of waves has been the focus of much previous work as many marine structures are (at least approximately) cylindrical, for example, the legs of a tension-leg or semi-submersible oil and gas platform. The linear diffraction problem for a single surface piercing truncated cylinder was solved by Garrett [6] using an eigenfunction expansion method and Yeung [7] solved the complementary radiation problems in surge, heave and pitch. The radiation and diffraction problems for a single submerged cylinder were solved by Jiang et al. [8,9].

The array diffraction problem has also been extensively studied. Kagemoto and Yue [10] combined features of the matrix method of Spring and Monkmeyer [11] and Simon [12], and the multiple scattering technique of Twersky [13] and Ohkusu [14] to solve the complete scattering problem for an array of arbitrary bodies. They represented the scattered wavefield around each body as a summation of cylindrical waves with undetermined amplitudes. A set of linear equations were derived to satisfy the diffraction characteristics of all the bodies. This system was then solved simultaneously for all of the unknown amplitude coefficients. The solution is in principle exact (within the context of linearised theory).

Linton and Evans [15] solved the diffraction problem for an array of $N$ bottom mounted cylinders using the method of Spring and Monkmeyer [11], and Kim [16] extended this to the complementary radiation problem. Yilmaz and Incecik [17] applied the interaction theory of Kagemoto and Yue [10] to the diffraction problem for truncated cylinders and Yilmaz [18] calculated the added mass and damping of an array of bodies oscillating as one. The present work is based on the methods outlined by Siddorn and Eatock Taylor [19], who investigated the wave excitation and response of surface-piercing truncated cylinders which are free to oscillate independently. They used the theory of Kagemoto and Yue [10], and developed an extension of this for the full radiation problem. In this work we adapt the method of Siddorn and Eatock Taylor [19] to consider submerged cylinders and produce results for a simple array. Array problems for different structures continue to be of interest - consider, for example, Chatjigeorgiou and Katsardi [20].

It is well known that linear solutions provide the basis for calculating mean drift loads in regular waves, which represent a simple, but sometimes important, non-linear effect. The second order mean drift forces on a two dimensional submerged horizontal cylinder were obtained by Ogilvie [21] using the first order solution and integrating the pressure over the surface. Lee and Newman [22] derived expressions for the vertical drift force on a submerged body assuming it is geometrically slender with respect to the body length, wavelength and submergence and using Kochin functions 
which avoid the need to determine the pressure distribution on the body surface. Mavrakos [23] solved for the mean vertical drift forces on axisymmetric bodies using conservation of momentum within a control volume. Here we solve for the second order mean vertical drift force by numerical pressure integration over the body surface as an example of further use of the model outputs in calculating hydrodynamic forces.

In Section 2 we present the diffraction and radiation solutions for a single submerged circular cylinder, followed by the transform matrix method to solve the system for an arbitrary array of cylinders of varying submergence, radius and thickness. In Section 3 the model is validated through comparison to the Hydrostar boundary element (BEM) software. Some limitations of the model and resonant behaviours above the cylinder are discussed, followed by array damping and exciting force results for a simple four cylinder array.

\section{Potential flow solutions for a submerged cylinder}

\subsection{Preliminaries}

We use linear potential flow theory; the wave amplitudes and body motions are small compared to the wavelength, device size and submergence. We use a velocity potential, denoted $\Phi$, throughout the fluid that varies sinusoidally with frequency $\omega$. The potential can be written in cylindrical coordinates as a complex Fourier series:

$$
\Phi=\Phi(r, \theta, z, t)=\operatorname{Re}\left\{\phi(r, \theta, z) \mathrm{e}^{\mathrm{i} \omega t}\right\}=\operatorname{Re}\left\{\mathrm{e}^{\mathrm{i} \omega t} \sum_{f=-\infty}^{\infty} \mathrm{e}^{\mathrm{i} f \theta} \chi_{f}(r, z)\right\} .
$$

Figure 2 defines the coordinate system, $t$ is time and $\phi$ is the complex time-invariant velocity potential. The angle invariant potential for each Fourier mode $f$ is denoted $\chi_{f}$. The fluid is divided into three regions, the core regions above and below the cylinder and an exterior region. The cylinder radius is $a$, the water depth $h$, the distance from the free surface to the top of the cylinder is submergence $s$ and the clearance between the sea floor and the bottom of the cylinder is $c$.
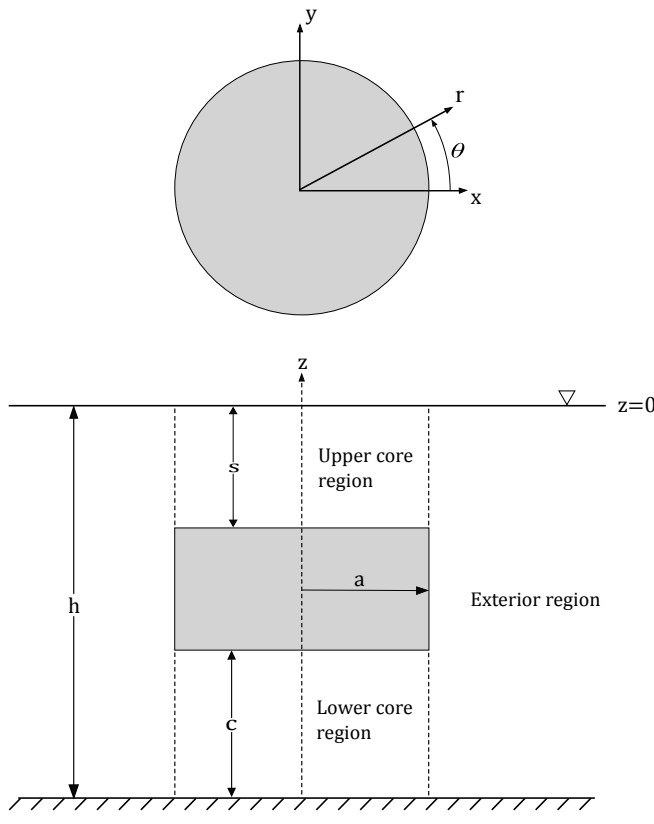

Figure 2: Definition sketch

The velocity potential must satisfy the Laplace equation in the fluid:

$$
\nabla^{2} \phi=0
$$


and the linearised boundary conditions on the seabed, free surface and cylinder surface $(S)$ respectively:

$$
\begin{gathered}
\left.\frac{\partial \phi}{\partial z}\right|_{z=-h}=0, \\
{\left[-\omega^{2} \phi+g \frac{\partial \phi}{\partial z}\right]_{z=0}=0,} \\
\left.\frac{\partial \phi}{\partial n}\right|_{S}=u_{n},
\end{gathered}
$$

where $u_{n}$ is the normal component of the complex amplitude of the cylinder velocity at any point on its surface. The dispersion relation arising from the free surface boundary condition (4) in finite depth is given by:

$$
\omega^{2}=g k_{m} \tanh \left(k_{m} h\right) .
$$

The positive real solution, $k_{0}$, is the wavenumber of the propagating mode. The negative imaginary solutions, $k_{m}$ for $m=1,2, \ldots$ are the wavenumbers of the evanescent modes. The evanescent waves are non-propagating modes, which decay exponentially with distance from the cylinder and as such cannot transfer energy into the far field.

\subsection{Potential solution in the exterior region}

A velocity potential in the exterior region which satisfies (2)-(4) is:

$$
\chi_{f}^{E}(r, z)=\sum_{m=0}^{\infty} Z_{m}(z)\left(A_{f m} \frac{P_{f m}\left(k_{m} r\right)}{P_{f m}\left(k_{m} a\right)}+a_{f m} \frac{Q_{f m}\left(k_{m} r\right)}{Q_{f m}\left(k_{m} a\right)}\right) .
$$

The remaining boundary condition (5) can be satisfied by choice of coefficients $A_{f m}$ and $a_{f m}$. The radial functions $P_{f m}\left(k_{m} r\right)$ representing outward travelling waves and $Q_{f m}\left(k_{m} r\right)$ representing incoming waves are defined by:

$$
P_{f m}\left(k_{m} r\right)=\left\{\begin{array}{ll}
H_{f}^{(2)}\left(k_{0} r\right) & m=0 \\
K_{f}\left(\mathrm{i} k_{m} r\right) & m=1,2, \ldots
\end{array} \quad Q_{f m}\left(k_{m} r\right)= \begin{cases}J_{f}\left(k_{0} r\right) & m=0 \\
I_{f}\left(\mathrm{i} k_{m} r\right) & m=1,2, \ldots\end{cases}\right.
$$

where $H_{f}^{(2)}=J_{f}-\mathrm{i} Y_{f}, K_{f}, J_{f}$ and $I_{f}$ are all Bessel functions in the standard notation. The exterior region velocity potential depth dependence is described by the function $Z_{m}(z)$ :

$$
Z_{m}(z)=\left(N_{m}^{E}\right)^{-1 / 2} \cosh \left(k_{m}(z+h)\right)
$$

where

$$
N_{m}^{E}=\frac{1}{2}\left[1+\frac{\sinh \left(2 k_{m} h\right)}{2 k_{m} h}\right]
$$

These functions are normalised so that

$$
\int_{-h}^{0}\left|Z_{m}(z)\right|^{2} \mathrm{~d} z=h
$$

\subsection{Upper core potential solution}

The potential in the upper core is split into the homogeneous and inhomogeneous solutions, $\chi_{f}^{U C}=\chi_{f}^{U C H}+\chi_{f}^{U C I}$. The inhomogeneous solution $\chi_{f}^{U C I}$ satisfies the boundary condition (5) for the cylinder oscillating and will be dealt with in the section on the radiation problem. The homogeneous solution $\chi_{f}^{U C H}$ satisfying the free surface condition (4) and the boundary condition (5) on the top surface of the cylinder for $\hat{u}_{n}=0$ can be written as:

$$
\chi_{f}^{U C H}(r, z)=\sum_{l=0}^{\infty} \beta_{f l} \frac{Q_{f l}\left(\lambda_{l} r\right)}{Q_{f l}\left(\lambda_{l} a\right)} U_{l}(z)
$$


where $\lambda_{l}$ are the real and imaginary solutions to the dispersion equation (6) substituting $s$ for $h$ and $\lambda_{l}$ for $k_{m}$. The functions $Q_{f l}\left(\lambda_{l} r\right)$ are defined in (8) and the coefficients $\beta_{f l}$ are unknown; $P_{f l}$ terms do not appear because they are unbounded as $r \rightarrow 0$. The functions $U_{l}(z)$ describe the depth dependence of the potential in the upper core and follow from equations (9) - (11) substituting $s$ for $h$ and $\lambda_{l}$ for $k_{m}$.

\subsection{Lower core potential solution}

The velocity potential in the lower core region is constructed differently, though the potential is again split into the homogeneous and inhomogeneous solutions $\chi_{f}^{L C}=\chi_{f}^{L C H}+\chi_{f}^{L C I}$. The homogeneous potential satisfying the seabed condition ( 3 ) and the body condition (5) for $u_{n}=0$, can be written as:

$$
\chi_{f}^{L C H}(r, z)=\frac{\alpha_{f 0}}{2}\left(\frac{r}{a}\right)^{|f|}+\sum_{n=1}^{\infty} \alpha_{f n} \frac{I_{f}\left(\lambda_{n} r\right)}{I_{f}\left(\lambda_{n} a\right)} \cos \left(\lambda_{n}(z+h)\right),
$$

where

$$
\lambda_{n}=\frac{n \pi}{c}, \quad n=1,2, \ldots .
$$

The coefficients $\alpha_{f n}$ are unknown and $n$ is the number of internal modes in the lower core.

\subsection{Diffraction problem}

To solve the diffraction problem we must determine the core coefficients $\alpha_{f n}$ and $\beta_{f l}$ as well as the exterior coefficients $A_{f m}$ for a given incident wave potential specified by $a_{f m}$. The homogeneous parts of the core potentials satisfy the boundary conditions on the cylinder top and bottom surfaces, while the boundary condition on the vertical sides of the cylinder is satisfied by the exterior $A_{f m}$ coefficients. By matching the potentials across the boundaries we can derive coupling equations that link the exterior and core coefficients; these form a system of equations that may be solved for each Fourier harmonic. Matching the exterior and core potentials across the boundary results in the following conditions:

$$
\begin{aligned}
\left.\chi_{f}^{L C}\right|_{r=a,-h \leq z \leq c-h} & =\left.\chi_{f}^{E}\right|_{r=a,-h \leq z \leq c-h}, \\
\left.\frac{\partial \chi_{f}^{L C}}{\partial r}\right|_{r=a,-h \leq z \leq c-h} & =\left.\frac{\partial \chi_{f}^{E}}{\partial r}\right|_{r=a,-h \leq z \leq c-h},
\end{aligned}
$$

with the same conditions applied in the upper core region for $r=a$ and $-s \leq z \leq 0$. The only remaining boundary condition is on the vertical sides of the cylinder:

$$
\left.\frac{\partial \chi_{f}^{E}}{\partial r}\right|_{r=a, c-h \leq z \leq-s}=0
$$

\subsubsection{Matching potentials}

We first link the lower core coefficients $\alpha_{f m}$ to the exterior coefficients $A_{f m}$ and $a_{f m}$. Using the orthogonality of $\cos \left(\lambda_{n}(z+h)\right)$ over the range $-h \leq z \leq c-h$ and applying the matching conditions in (15) at the boundary we find:

$$
\alpha_{f n}=\sum_{m=0}^{\infty}\left(A_{f m}+a_{f m}\right) C_{n m},
$$

where $C_{n m}$ is a coupling integral between the depth dependence in the lower core and exterior regions:

$$
\begin{aligned}
C_{n m} & =\frac{2}{c} \int_{-h}^{c-h} \cos \left(\lambda_{n}(z+h)\right) Z_{m}(z) \mathrm{d} z \\
& =\frac{2}{c}\left(N_{m}^{E}\right)^{-1 / 2} \begin{cases}\sinh \left(k_{m} c\right) / k_{m} & n=0 \\
\frac{k_{m}(-1)^{n} \sinh \left(k_{m} c\right)}{\lambda_{n}^{2}+k_{m}^{2}} & n=1,2, \ldots,\end{cases}
\end{aligned}
$$


In a similar manner we can derive an expression linking the upper core coefficients $\beta_{f l}$ to the exterior coefficients. Using the orthogonality of $U_{l}(z)$ over the region $-s \leq z \leq 0$ then applying the matching condition (15):

$$
\beta_{f l}=\sum_{m=0}^{\infty}\left(A_{f m}+a_{f m}\right) G_{l m},
$$

where, similarly, $G_{l m}$ couples the depth dependence in the exterior and upper core regions:

$$
G_{l m}=\frac{\left(N_{l}^{T} N_{m}^{E}\right)^{-1 / 2}}{s}\left(\frac{k_{m} \cosh \left(\lambda_{l} s\right) \sinh \left(k_{m} h\right)-k_{m} \sinh \left(k_{m}(h-s)\right)-\lambda_{l} \cosh \left(k_{m} h\right) \sinh \left(\lambda_{l} s\right)}{k_{m}^{2}-\lambda_{l}^{2}}\right)
$$

The third key equation is found using the orthogonality of $Z_{m}(z)$ over the range $-h \leq z \leq 0$. Differentiating with respect to $r$ at $r=a$ and splitting the integral over the depth, leads to:

$$
\sum_{n=0}^{\infty} \alpha_{f n} S_{f n} C_{n m}+\sum_{l=0}^{\infty} \beta_{f l} V_{f l} G_{l m}=A_{f m} \frac{P_{f m}^{\prime}\left(k_{m} a\right)}{P_{f m}\left(k_{m} a\right)}+a_{f m} \frac{Q_{f m}^{\prime}\left(k_{m} a\right)}{Q_{f m}\left(k_{m} a\right)}
$$

where the primes indicate radial derivatives. $S_{f n}$ and $V_{f l}$ are given by:

$$
\begin{gathered}
S_{f n}= \begin{cases}|f| c / 4 a h & n=0 \\
\frac{c}{2 h} \lambda_{n} \frac{I_{f}^{\prime}\left(\lambda_{n} a\right)}{I_{f}\left(\lambda_{n} a\right)} & n=1,2, \ldots,\end{cases} \\
V_{f l}=\frac{s}{h} \frac{Q_{f l}^{\prime}\left(\lambda_{l} a\right)}{Q_{f l}\left(\lambda_{l} a\right)} .
\end{gathered}
$$

The infinite sums in equations (17), (19) and (21) are truncated to include $M$ evanescent modes, $N$ lower core modes and $L$ upper core modes. For any given value of Fourier harmonic $f$ they can be written as matrix equations:

$$
\begin{gathered}
\alpha=M_{1} \mathbf{A}+M_{1} \mathbf{a}, \\
\beta=M_{2} \mathbf{A}+M_{2} \mathbf{a}, \\
M_{3} \alpha+M_{4} \beta=M_{5} \mathbf{A}+M_{6} \mathbf{a} .
\end{gathered}
$$

The column vectors a and $\mathbf{A}$ are of size $\mathbf{M}$, and column vectors $\alpha$ and $\beta$ are of size $N$ and $L$ respectively. The matrices $M_{1}, \ldots, M_{6}$ are defined by:

$$
\begin{aligned}
& M_{1}=\left\{C_{n m}\right\} \\
& M_{2}=\left\{G_{l m}\right\} \\
& M_{3}=\left\{C_{n m}\right\}^{T} * \operatorname{diag}\left(S_{n}\right) \\
& M_{4}=\left\{G_{l m}\right\}^{T} * \operatorname{diag}\left(V_{l}\right) \\
& M_{5}=\operatorname{diag}\left(\frac{P_{m}^{\prime}\left(k_{m} a\right)}{P_{m}\left(k_{m} a\right)}\right) \\
& M_{6}=\operatorname{diag}\left(\frac{Q_{m}^{\prime}\left(k_{m} a\right)}{Q_{m}\left(k_{m} a\right)}\right)
\end{aligned}
$$

where "diag" indicates matrix diagonalisation of the vector, i.e. $\operatorname{diag}\left(a_{1}, \ldots, a_{n}\right)=\left[\begin{array}{lll}a_{1} & & \\ & \ddots & \\ & & a_{n}\end{array}\right]$ and the superscript $T$ is used to denote transpose. 


\subsection{Radiation problem}

We allow the cylinder to move in any combination of six degrees of freedom; surge, sway, heave, roll, pitch and yaw (denoting the complex velocity amplitudes $u_{1}, \ldots, u_{6}$ respectively). The boundary condition (5) on the surface of the cylinder is satisfied by the inhomogeneous potentials in the core regions and by choice of the $A_{f m}$ coefficients in the exterior region. The linearised boundary condition on the bottom of the cylinder is:

$$
\left.\frac{\partial \chi_{f}^{L C I}}{\partial z}\right|_{z=c-h, 0 \leq r \leq a}= \begin{cases}u_{3} & f=0, \\ -\frac{1}{2} u_{5} r+\frac{1}{2} f \mathrm{i} u_{4} r & f= \pm 1 \\ 0 & |f| \geq 2 .\end{cases}
$$

A velocity potential in the lower core region that satisfies this boundary condition along with the Laplace equation (2) and the seabed boundary condition (3) follows from Yeung [7]:

$$
\chi_{f}^{L C I}= \begin{cases}u_{3} \times \frac{1}{2}\left((z+h)^{2}-r^{2} / 2\right) / c & f=0, \\ \left(-\frac{1}{2} u_{5}+\frac{1}{2} f i u_{4}\right) \times \frac{1}{2}\left((z+h)^{2} r-r^{3} / 4\right) / c & f= \pm 1, \\ 0 & |f| \geq 2 .\end{cases}
$$

On the vertical sides of the cylinder the boundary condition (16) satisfied by the exterior potential becomes:

$$
\left.\frac{\partial \chi_{f}^{E}}{\partial r}\right|_{r=a, c-h \leq z \leq-s}= \begin{cases}\frac{1}{2} u_{1}-\frac{1}{2} f \mathrm{i} u_{2}+\frac{1}{2} u_{5}\left(z-z_{0}\right)-\frac{1}{2} f \mathrm{i} u_{4}\left(z-z_{0}\right) & f= \pm 1, \\ 0 & f \neq \pm 1,\end{cases}
$$

where the rotational motion of the cylinder is assumed to be around the point $\left(0,0, z_{0}\right)$. The linearised boundary condition on the upper surface of the cylinder is the same as that on the lower surface (28) except evaluated at $z=-s$. A velocity potential in the upper core satisfying the body surface boundary condition, the free surface boundary condition (4) and the Laplace equation (2) follows from McIver and Evans [4]:

$$
\chi_{f}^{U C I}= \begin{cases}u_{3} \times\left(z+g / \omega^{2}\right) & f=0, \\ \left(-\frac{1}{2} u_{5}+\frac{1}{2} f \mathrm{i} u_{4}\right) \times r\left(z+g / \omega^{2}\right) & f= \pm 1, \\ 0 & |f| \geq 2 .\end{cases}
$$

This form of the velocity potential is simpler than that used by Jiang et al. [9].

\subsubsection{Solving for $A^{*}, \alpha^{*}$ and $\beta^{*}$}

The inhomogeneous solutions in the core regions introduce the additional components $A^{*}, \alpha^{*}$ and $\beta^{*}$ to the key equations (17), (19) and (21). We integrate the inhomogeneous potentials in the core regions and the boundary condition on the cylinder sides over the depth and apply the matching conditions in equation (15):

$$
\begin{gathered}
A_{f m}^{*}=\left.\frac{1}{h} \int_{-h}^{c-h} \frac{\partial \chi_{f}^{L C I}}{\partial r}\right|_{r=a} Z_{m}(z) \mathrm{d} z+\left.\frac{1}{h} \int_{c-h}^{-s} \frac{\partial \chi_{f}^{E}}{\partial r}\right|_{r=a} Z_{m}(z) \mathrm{d} z+\left.\frac{1}{h} \int_{-s}^{0} \frac{\partial \chi_{f}^{U C I}}{\partial r}\right|_{r=a} Z_{m}(z) \mathrm{d} z, \\
\alpha_{f n}^{*}=\left.\frac{2}{c} \int_{-h}^{c-h} \chi_{f}^{L C I}\right|_{r=a} \cos \left(\lambda_{n}(z+h)\right) \mathrm{d} z, \\
\beta_{f l}^{*}=\left.\frac{1}{s} \int_{-s}^{0} \chi_{f}^{U C I}\right|_{r=a} U_{l}(z) \mathrm{d} z .
\end{gathered}
$$

For any value of $f$, we can write $A_{f m}^{*}$ as a column vector $A_{f}^{*}=A_{f}^{\prime} \mathbf{u}$, where $A_{f}^{\prime}$ is a matrix with $M$ rows and 6 columns. Similarly we can write $\alpha_{f n}^{*}$ as a column vector $\alpha_{f}^{*}=\alpha_{f}^{\prime} \mathbf{u}$. Including contributions from the inhomogeneous core potentials, the three key equations (24)-(26) now become:

$$
\alpha+\alpha^{\prime} \mathbf{u}=M_{1} \mathbf{A}+M_{1} \mathbf{a}
$$




$$
\begin{gathered}
\beta+\beta^{\prime} \mathbf{u}=M_{2} \mathbf{A}+M_{2} \mathbf{a}, \\
M_{3} \alpha+M_{4} \beta+A^{\prime} \mathbf{u}=M_{5} \mathbf{A}+M_{6} \mathbf{a} .
\end{gathered}
$$

This system of equations can be solved for the velocity potential coefficients $\mathbf{A}, \alpha$ and $\beta$. Solving simultaneous equations we find:

$$
\begin{aligned}
\mathbf{A} & =\left(M_{3} M_{1}+M_{4} M_{2}-M_{5}\right)^{-1}\left(\left(M_{6}-M_{3} M_{1}-M_{4} M_{2}\right) \mathbf{a}-\left(A^{\prime}-M_{3} \alpha^{\prime}+M_{4} \beta^{\prime}\right) \mathbf{u}\right) \\
& =B^{E} \mathbf{a}+D^{E} \mathbf{u} \\
\alpha & =\left(M_{1} B^{E}+M_{1}\right) \mathbf{a}+\left(M_{1} D^{E}-\alpha^{\prime}\right) \mathbf{u} \\
& =B^{L C} \mathbf{a}+D^{L C} \mathbf{u} \\
\beta & =\left(M_{2} B^{E}+M_{2}\right) \mathbf{a}+\left(M_{2} D^{E}-\beta^{\prime}\right) \mathbf{u} \\
& =B^{U C} \mathbf{a}+D^{U C} \mathbf{u} .
\end{aligned}
$$

These equations are valid for any value of $f$. Matrices $B^{E}, B^{L C}$ and $B^{U C}$ are the exterior, lower core and upper core diffraction matrices respectively, while $D^{E}, D^{L C}$ and $D^{U C}$ are the analogous radiation matrices.

\subsection{Multiple scattering by an array}

The radiation and diffraction problem for a single cylinder has now been fully formulated and we turn our attention to the same problem for an arbitrary array of submerged cylinders. The multiple scattering technique here follows Siddorn and Eatock Taylor [19] and Yilmaz [18]. We assume that all cylinders have the same geometry and submergence, though this is not necessary for the method to apply. We define a local coordinate system centred on each cylinder as in figure 3.

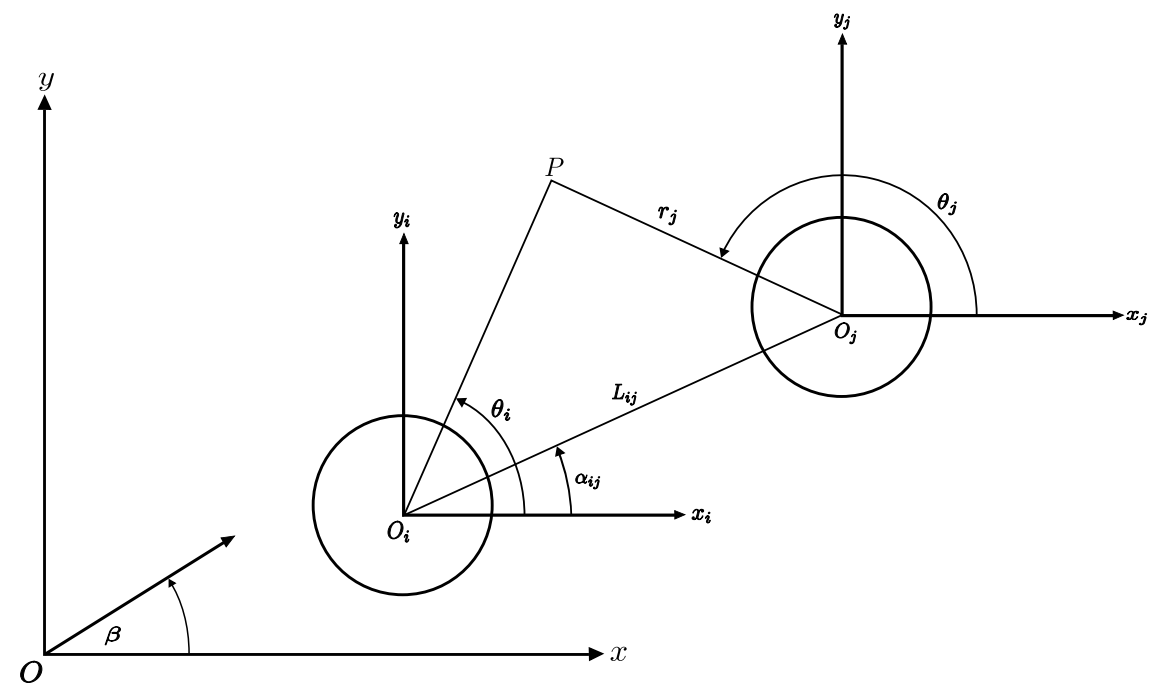

Figure 3: Plan view of two cylinders and coordinate system after Yilmaz and Incecik [17]

To solve the scattering problem we use a transform matrix to relate an outgoing wave from cylinder $i$ to an incoming wave on cylinder $j$. We define local coordinates for cylinder $i$ as $\left(r_{i}, \theta_{i}\right)$ and $\left(r_{j}, \theta_{j}\right)$ for cylinder $j$. We equate an outgoing wave exterior potential to an incoming wave exterior potential from (7):

$$
\sum_{f=-\infty}^{\infty} \mathrm{e}^{\mathrm{i} f \theta_{i}} \sum_{m=0}^{\infty} A_{f m}^{i} \frac{P_{f m}\left(k_{m} r_{i}\right)}{P_{f m}\left(k_{m} a\right)} Z_{m}(z)=\sum_{f=-\infty}^{\infty} \mathrm{e}^{\mathrm{i} f \theta_{j}} \sum_{m=0}^{\infty} a_{f m}^{j} \frac{Q_{f m}\left(k_{m} r_{j}\right)}{Q_{f m}\left(k_{m} a\right)} Z_{m}(z) .
$$

Graf's addition theorem for Bessel functions can be utilised for the coordinate transform between cylinders (see Abramowitz and Stegun [24] eq. 9.1.79), allowing us to relate the functions $P_{f m}\left(k_{m} r_{i}\right)$ and $Q_{f m}\left(k_{m} r_{j}\right)$. We can thus 
define the transform matrix $T_{m f n}^{i j}$ for the evanescent and propagating waves:

$$
\frac{P_{n m}\left(k_{m} r_{i}\right)}{P_{n m}\left(k_{m} a\right)} \mathrm{e}^{\mathrm{i} n \theta_{i}}=\sum_{f=-\infty}^{\infty} T_{m f n}^{i j} \frac{Q_{f m}\left(k_{m} r_{j}\right)}{Q_{f m}\left(k_{m} a\right)} \mathrm{e}^{\mathrm{i} f \theta_{j}},
$$

where

$$
T_{m f n}^{i j}= \begin{cases}H_{n-f}^{(2)}\left(k_{0} L_{i j}\right) \mathrm{e}^{\mathrm{i} \alpha_{i j}(n-f)} Q_{f 0}\left(k_{0} a\right) / P_{n 0}\left(k_{0} a\right) & m=0 \\ K_{n-f}\left(\mathrm{i} k_{m} L_{i j}\right) \mathrm{e}^{\mathrm{i} \alpha_{i j}(n-f)}(-1)^{f} Q_{f m}\left(k_{m} a\right) / P_{n m}\left(k_{m} a\right) & m=1,2, \ldots\end{cases}
$$

Substituting the transform relation (42) into (41) and equating each term of the Fourier series we obtain:

$$
a_{f m}^{j}=\sum_{n=-\infty}^{\infty} T_{m f n}^{i j} A_{n m}^{i}
$$

The Fourier series over $f$ and the infinite sum in $n$ are truncated to $F$ terms. We write $a_{f m}^{j}$ and $A_{n m}^{i}$ as column vectors $\mathbf{a}_{m}^{j}(f)$ and $\mathbf{A}_{m}^{i}(n)$, each of size $F . T_{m}^{i j}$ is defined as the $F \times F$ matrix formed by the terms $T_{m}^{i j}(f, n)=T_{m f n}^{i j}$. The transform equation (44) can then be written in matrix form as:

$$
\mathbf{a}_{m}^{j}=T_{m}^{i j} \mathbf{A}_{m}^{i}
$$

The transform matrix equation (45) and the single cylinder matrix equations (38), (39) and (40) can be solved to determine the coefficients for the complete radiation and diffraction problem for an array of cylinders. To extend the single cylinder equations to incorporate multiple scattering we define a full vector form of the column vectors $\alpha$, A, a and $\beta$, containing all values of cylinder index $j$, Fourier harmonic $f$ and wave mode number $m$. We denote the full column vector form of the variables by $\hat{\mathbf{u}}, \hat{\mathbf{a}}, \hat{\mathbf{A}}, \hat{\alpha}, \hat{\beta}$ and $\hat{\mathbf{a}}^{0}$. We can now write the full form of the diffraction, radiation and coordinate transform matrices. These are denoted by $\hat{B}^{E}, \hat{D}^{E}, B^{\hat{U} C}, \hat{B}^{L C}, \hat{D}^{U C}, \hat{D}^{L C}$ and $\hat{T}$. The radiation and diffraction matrices are all block diagonal, where each block represents the coefficients of a single cylinder.

\subsection{Combining equations}

Using the full vectors we can write the following equations:

$$
\begin{aligned}
\hat{\mathbf{a}} & =\hat{T} \hat{\mathbf{A}}, \\
\hat{\mathbf{A}} & =\hat{D}^{E} \hat{\mathbf{u}}+\hat{B}^{E}\left(\hat{\mathbf{a}}+\hat{\mathbf{a}}^{0}\right), \\
\hat{\alpha} & =\hat{D}^{L C} \hat{\mathbf{u}}+\hat{B}^{L C}\left(\hat{\mathbf{a}}+\hat{\mathbf{a}}^{0}\right), \\
\hat{\beta} & =\hat{D}^{U C} \hat{\mathbf{u}}+\hat{B}^{U C}\left(\hat{\mathbf{a}}+\hat{\mathbf{a}}^{0}\right) .
\end{aligned}
$$

where $\hat{\mathbf{a}}$ is the coefficient vector of incident waves which are caused by scattering from the array and $\hat{\mathbf{a}}^{0}$ is the coefficient vector of an ambient incident wave which comes from outside the array. The sum of these two coefficients is the total incident wave on each cylinder. Solving the simultaneous equations:

$$
\begin{aligned}
\hat{A} & =\left(I-\hat{B}^{E} \hat{T}\right)^{-1}\left(\hat{D}^{E} \hat{\mathbf{u}}+\hat{B}^{E} \hat{\mathbf{a}}^{0}\right) \\
& =X\left(\hat{D}^{E} \hat{\mathbf{u}}+\hat{B}^{E} \hat{\mathbf{a}}^{0}\right)
\end{aligned}
$$

The effect of multiple scattering can be described by the the matrix $X=\left(I-\hat{B}^{E} \hat{T}\right)^{-1}$. The coefficients of the total incident wave on each cylinder are given by:

$$
\begin{aligned}
\hat{\mathbf{a}}^{t o t} & =\hat{\mathbf{a}}^{0}+\hat{\mathbf{a}} \\
& =\hat{\mathbf{a}}^{0}+\hat{T} X\left(\hat{D}^{E} \hat{\mathbf{u}}+\hat{B}^{E} \hat{\mathbf{a}}^{0}\right) \\
& =\left(I+\hat{T} X \hat{B}^{E}\right) \hat{\mathbf{a}}^{0}+\hat{T} X \hat{D}^{E} \hat{\mathbf{u}}
\end{aligned}
$$


and the coefficients of the core potentials are given by:

$$
\begin{aligned}
\hat{\alpha} & =\hat{D}^{L C} \hat{\mathbf{u}}+\hat{B}^{L C}\left(\hat{\mathbf{a}}+\hat{\mathbf{a}}^{0}\right) \\
& =\left(\hat{B}^{L C}+\hat{B}^{L C} \hat{T} X \hat{B}^{E}\right) \hat{\mathbf{a}}^{0}+\left(\hat{B}^{L C} \hat{T} X \hat{D}^{E}+\hat{D}^{L C}\right) \hat{\mathbf{u}},
\end{aligned}
$$

similarly,

$$
\hat{\beta}=\left(\hat{B}^{U C}+\hat{B}^{U C} \hat{T} X \hat{B}^{E}\right) \hat{\mathbf{a}}^{0}+\left(\hat{B}^{U C} \hat{T} X \hat{D}^{E}+\hat{D}^{U C}\right) \hat{\mathbf{u}} .
$$

This completes the solution of the combined radiation and diffraction problem for an array of submerged cylinders. The coefficients of the velocity potential in both the exterior and core regions have been determined.

\subsection{Free surface, forces and moments}

Now that the velocity potential is known we can evaluate the free surface elevation at any point in the exterior and upper core:

$$
\eta(r, \theta, t)=-\left.\frac{1}{g} \frac{\partial \Phi(r, \theta, z, t)}{\partial t}\right|_{z=0}=-\left.\operatorname{Re}\left\{\frac{\mathrm{i} \omega}{g} \phi(r, \theta, z) \mathrm{e}^{\mathrm{i} \omega t}\right\}\right|_{z=0} .
$$

For each cylinder, the diffraction and radiation forces acting on the cylinder can be found by integrating the pressure $p=\operatorname{Re}\left\{\hat{p} e^{i \omega t}\right\}$ over the surface, where

$$
\hat{p}=-\mathrm{i} \omega \rho \phi,
$$

where $\rho$ is the fluid density. The heave force is found by integrating the pressure on the bottom and top surfaces of the cylinder using the homogeneous and inhomogeneous core potentials. The complex amplitude of the heave force due to the upper core homogeneous potential is:

$$
\begin{aligned}
\frac{\hat{F}_{3}^{U C H}}{\mathrm{i} \omega \rho} & =\left.\int_{0}^{a} \int_{0}^{2 \pi} \phi\right|_{z=-s} r \mathrm{~d} \theta \mathrm{d} r \\
& =\int_{0}^{a} \int_{0}^{2 \pi} \sum_{f=-\infty}^{\infty} \mathrm{e}^{\mathrm{i} f \theta} \sum_{l=0}^{\infty} \beta_{f l} \frac{Q_{f l}\left(\lambda_{l} r\right)}{Q_{f l}\left(\lambda_{l} a\right)}\left(N_{l}^{T}\right)^{-1 / 2} r \mathrm{~d} r \mathrm{~d} \theta,
\end{aligned}
$$

and the integral can be evaluated as:

$$
\frac{\hat{F}_{3}^{U C H}}{\mathrm{i} \omega \rho \pi a^{2}}=\frac{2\left(N_{l}^{T}\right)^{-1 / 2}}{a}\left(\frac{\beta_{00}}{\lambda_{0}} \frac{J_{1}\left(\lambda_{0} a\right)}{J_{0}\left(\lambda_{0} a\right)}+\sum_{l=1}^{\infty} \frac{\beta_{0 l}}{\mathrm{i} \lambda_{l}} \frac{I_{1}\left(\mathrm{i} \lambda_{l} a\right)}{I_{0}\left(\mathrm{i} \lambda_{l} a\right)}\right) .
$$

The force due to the upper core inhomogeneous potential is:

$$
\frac{\hat{F}_{3}^{U C I}}{\mathrm{i} \omega \rho}=\int_{0}^{a} \int_{0}^{2 \pi} u_{3}\left(\frac{g}{\omega^{2}}-s\right) r \mathrm{~d} \theta \mathrm{d} r,
$$

which can be evaluated as:

$$
\frac{\hat{F}_{3}^{U C I}}{\mathrm{i} \omega \rho \pi a^{2}}=u_{3}\left(\frac{g}{\omega^{2}}-s\right) .
$$

The lower core is treated similarly, and the complex amplitude of the total heave force is found by summing the components from the upper and lower homogeneous and inhomogeneous core potentials:

$$
\begin{aligned}
& \frac{\hat{F}_{3}}{\mathrm{i} \omega \rho \pi a^{2}}=-\left(\frac{\alpha_{00}}{2}+\frac{2}{a} \sum_{n=1}^{\infty} \frac{\alpha_{0 n}}{\lambda_{n}} \frac{I_{1}\left(\lambda_{n} a\right)}{I_{0}\left(\lambda_{n} a\right)}(-1)^{n}\right)-u_{3}\left(\frac{c}{2}-\frac{a^{2}}{8 c}\right)+ \\
& \frac{2\left(N_{l}^{T}\right)^{-1 / 2}}{a}\left(\frac{\beta_{00}}{\lambda_{0}} \frac{J_{1}\left(\lambda_{0} a\right)}{J_{0}\left(\lambda_{0} a\right)}+\sum_{l=1}^{\infty} \frac{\beta_{0 l}}{\mathrm{i} \lambda_{l}} \frac{I_{1}\left(\mathrm{i} \lambda_{l} a\right)}{I_{0}\left(\mathrm{i} \lambda_{l} a\right)}\right)+u_{3}\left(\frac{g}{\omega^{2}}-s\right) .
\end{aligned}
$$


The surge force, sway force, pitch moment and roll moment calculations are given in Appendix A. For an axisymmetric device the yaw moment is always zero.

\subsection{Radiation impedance and excitation force}

For a system of $J$ cylinders we define the full force vector $\mathbf{F}$. This is evaluated with the matrix equation:

$$
\mathbf{F}=F_{A}\left(\hat{\mathbf{A}}+\hat{\mathbf{a}}^{t o t}\right)+F_{\alpha} \hat{\alpha}+F_{\beta} \hat{\beta}+F_{u} \hat{\mathbf{u}}
$$

where $F_{A}, F_{\alpha}, F_{\beta}$ and $F_{u}$ are defined using equation (56) and similar expressions for the other force components. Using the standard approach we can write this as the sum of an exciting force and radiation force. The exciting force vector is given by:

$$
\begin{aligned}
\mathbf{F}_{e} & =\left[F_{A}\left(X \hat{B}^{E}+I+\hat{T} X \hat{B}^{E}\right)+F_{\alpha}\left(B^{L C}+\hat{B}^{L C} \hat{T} X \hat{B}^{E}\right)+F_{\beta}\left(\hat{B}^{U C}+\hat{B}^{U C} \hat{T} X \hat{B}^{E}\right)\right] \hat{\mathbf{a}}^{0} \\
& =E \hat{\mathbf{a}}^{0}
\end{aligned}
$$

The radiation force vector is given by:

$$
\begin{aligned}
\mathbf{F}_{z} & =\left[F_{A}\left(X \hat{D}^{E}+\hat{T} X \hat{D}^{E}\right)+F_{\alpha}\left(\hat{B}^{L C} \hat{T} X \hat{D}^{E}+\hat{D}^{L C}\right)+F_{\beta}\left(\hat{B}^{U C} \hat{T} X \hat{D}^{E}+\hat{D}^{U C}\right)+F_{u}\right] \hat{\mathbf{u}} \\
& =-(B+\mathrm{i} \omega A) \hat{\mathbf{u}},
\end{aligned}
$$

where $B$ is the damping matrix and $A$ is the added mass matrix, both square matrices of size $6 J$ where $J$ is the number of cylinders.

\section{Results}

\subsection{Comparison with BEM}

We compare sample force and surface elevation data with results generated using the commercial boundary element model HydroStar [25]. Figure 4 shows the added mass, damping (diagonal terms) and exciting force for the up-wave body in an array of two cylinders separated by $4 a$ and oriented for head seas (wave crest perpendicular to the line joining the cylinders) with cylinder geometry $T / a=0.3$ (where $T$ is the cylinder thickness), $s / h=0.06$ and $h / a=5$. The results show excellent agreement for the frequency range considered. To achieve adequate convergence the number of modes was increased until a variation of less than $1 \%$ was observed. Note that here we are interested in a submerged cylinder somewhat resembling a CETO device, which is very much closer to the free surface than to the seabed. This allows us to use informed judgement when choosing the ratios of numbers of evanescent modes used in the different regions. Considering the form of (20), where large coupling will occur if the denominator is small, and noting that evanescent wave-numbers for the outer region approach $-\mathrm{i} M \pi / h$ and for the upper core region approach $-\mathrm{i} L \pi / s$ as the number of modes is increased, we can say that the largest coupling will occur when $M \approx L h / s$. Thus we should choose numbers of evanescent modes $M, L$ approximately in the ratio $h: s$. For body forces adequate convergence was found with $F=9$ Fourier modes, $M=39$ exterior modes $L=5$ upper core modes and $N=35$ lower core modes. Figure 5a shows the surface elevation considering only the diffracted wave at a point between two cylinders (off the centre line) for head seas. For this point convergence of the diffracted surface elevation was found for $F=11, M=26, L=3$ and $N=12$ modes. Figures $5 \mathrm{~b}$ and $5 \mathrm{c}$ show the surface elevation at one point above two cylinders, where only one cylinder is oscillating and there are no incident waves. For these points convergence in surface elevation was found for $F=11, M=40, L=4$ and $N=19$ in heave and $F=11, M=108, L=11$ and $N=26$ in pitch. 


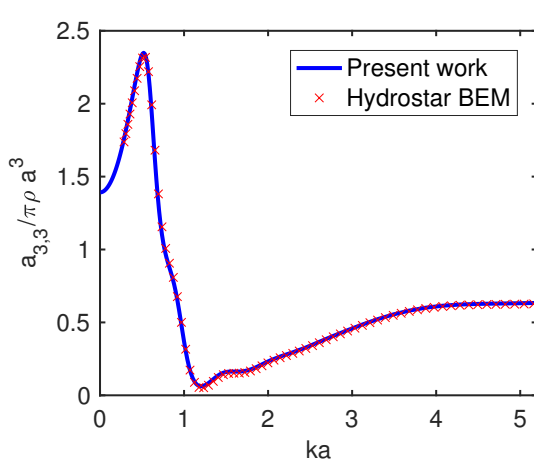

(a) Added mass

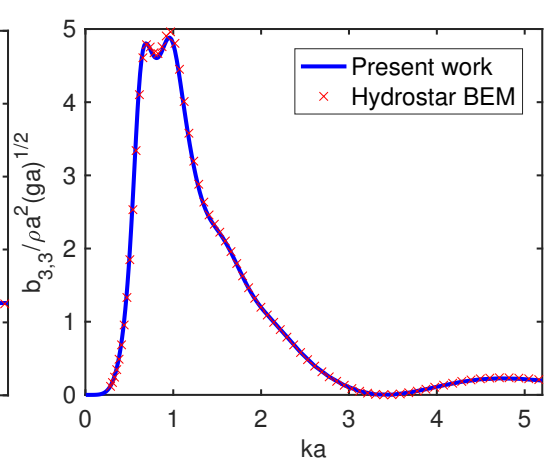

(b) Damping

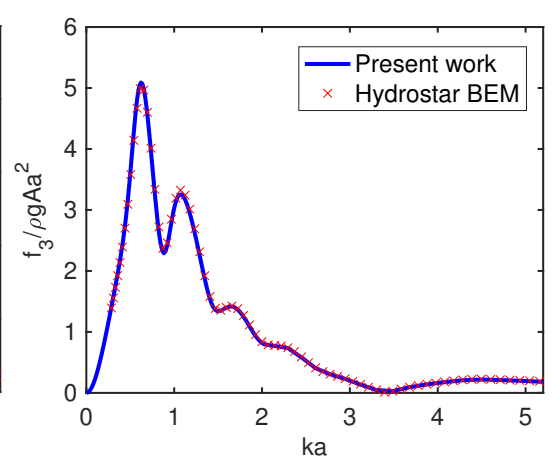

(c) Exciting force

Figure 4: Added mass, damping and modulus exciting force in heave for the up-wave body in an array of two submerged cylinders, spacing $4 a$, $\beta=0$ (head seas) thickness $T / a=0.3$, submergence $s / h=0.06$ and water depth $h / a=5$

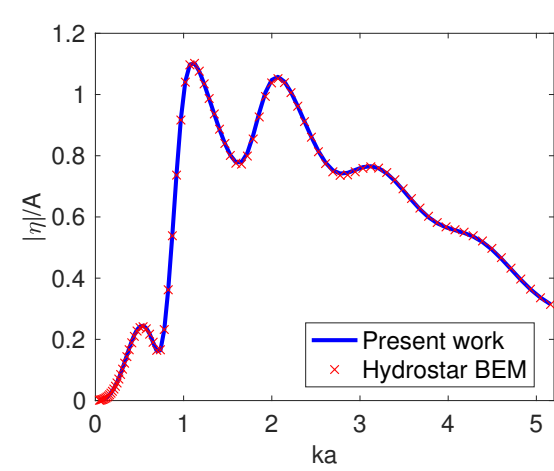

(a) Diffraction

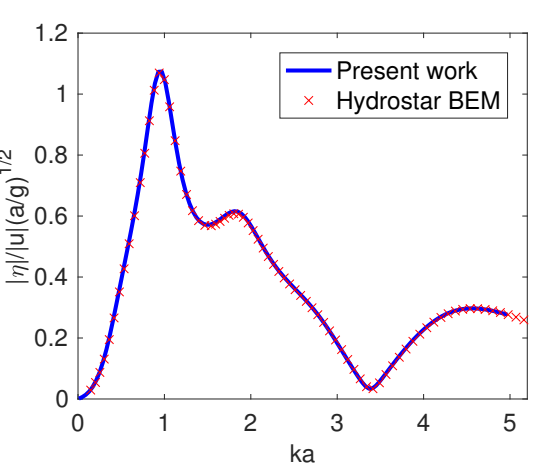

(b) Heave

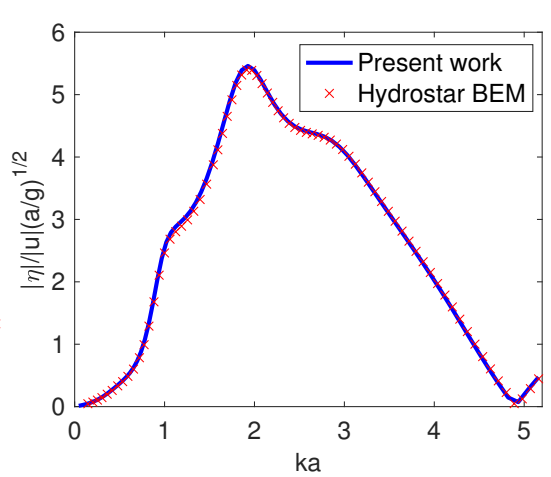

(c) Pitch

Figure 5: (a) Amplitude of diffracted surface elevation (minus incident wave) at $x=0, y / a=1$ for two cylinders at $x= \pm 2 a, y=0$. (b,c) Surface elevation at point $x / a=0, y=0$ in heave and pitch above two submerged cylinders located at $x= \pm 2 a, y=0$, radiated wave for one cylinder oscillating only, thickness $T / a=0.3$, submergence $s / h=0.06$ and water depth $h / a=5$.

\subsection{Resonances above the cylinder}

The region of fluid above a shallowly submerged cylinder is known to support resonances, which might be described as sloshing resonances (McIver and McIver [26]), in that they may be regarded as being associated with a pole in the velocity potential near the real axis in complex frequency space. Such resonances are associated with large motions of the free surface and, where they couple with rigid body modes, rapid variation in the added mass and damping coefficients (and hence exciting force). Such resonances have been studied for simple submerged heaving cylinders by, for example, Newman et al. [3] in 2D and McIver and Evans [4] for an axisymmetric submerged heaving cylinder that extends to the sea floor. The present model can be used to predict when such resonances occur (according to linear theory) which is valuable as these resonances are the dominant effect for shallowly submerged cylinders. In this work the resonances are characterised for a fixed geometry cylinder with varying submergence $(s)$ in frequency space. We consider the radiation problem and choose to characterise the resonances by amplification of the free surface above the cylinder, as in figure 6a for the heave problem. The bands of large surface elevation represent individual resonant modes, each with their own spatial variation. As identified by McIver and Evans [4], maximum surface elevation and radiation damping will both be associated with large radial flow at the cylinder edge, and in the limit $s \rightarrow 0$ (where evanescent modes are not significant), this will occur near $J_{0}\left(\lambda_{0} a\right)=0$ for heave. Somewhat like the modes in a cylindrical moonpool (though with different boundary conditions), these axisymmetric mode shapes are approximately defined by $J_{0}$, the first mode corresponding to a single peak (anti-node) above the cylinder and the second to out of phase anti-nodes in the upper core region. As the added mass and damping are derived using the pressure integrated 
over the cylinder surface, the effect of the second and higher modes on these hydrodynamic coefficients is small. For heave motion the problem is radially symmetric and the maximum surface elevation is always above the centre of the cylinder. At such shallow submergences the resonant fluid motion above the cylinder is extremely large compared to the stroke $\left(\zeta_{n}=u_{n} /(i \omega)\right)$.

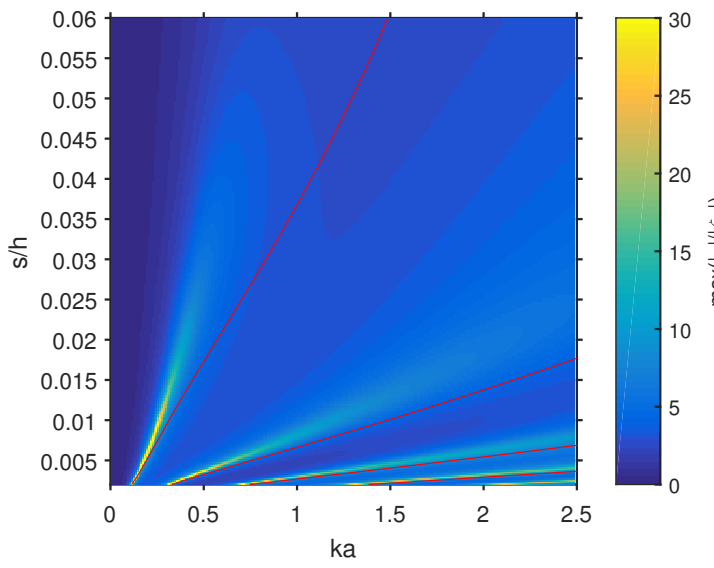

(a) Heave

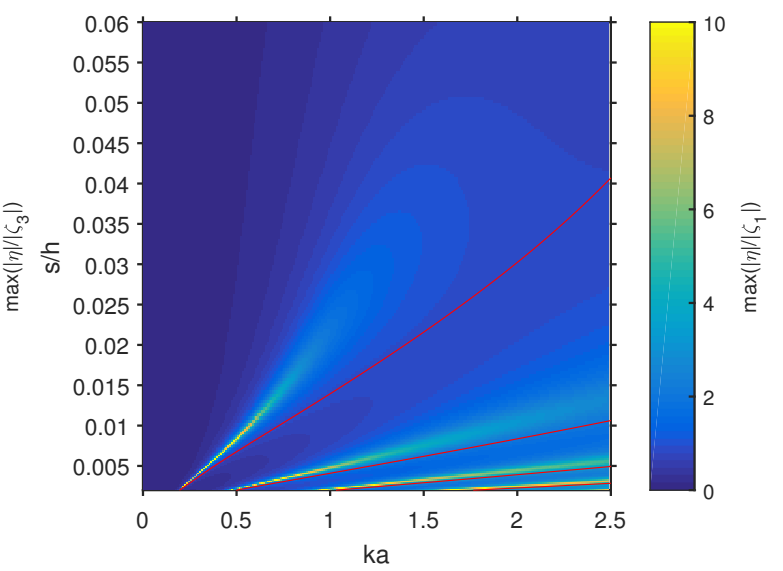

(b) Surge

Figure 6: Maximum surface elevation (indicated by colour) in the upper core region above a single cylinder, radiated wave only, $h / a=5, T / a=0.3$. (a) heave, (b) surge where the red lines indicate zeros of the Bessel function $J_{0}\left(\lambda_{0} a\right)$ and $J_{1}\left(\lambda_{0} a\right)$ respectively.

Figure $6 \mathrm{~b}$ shows the maximum surface elevation above a cylinder moving in surge only, plotted in submergencefrequency space. We see resonant bands occurring as in the heave case, however the surge radiated wave is no longer radially symmetric. Linear theory predicts large amplitude waves in the upper core (at resonance, over 10 times the stroke) for shallow submergences, an interesting phenomenon given that the cylinder is not moving vertically.

It is clear from the limited results presented above that the resonances dominate behaviour of the cylinder in waves and that the associated free surface elevations are very large. It is well known that potential flow models do not reflect real fluid behaviour around such resonances unless the amplitude is suitably small - in this case, compared to the submergence of the cylinder. It is possible to apply an artificial damping term to the fluid to reduce the effect of resonances; for example, van Winsen et al. [27] investigated the application of a damping lid to the diffraction problem for a shallowly submerged cylinder in an attempt to improve the accuracy of heave force calculations. Numerous authors have approached the problem of modelling non-linear flow above a shallowly submerged body. Grue [28] used the Boussinesq equations coupled to potential flow while Ertekin and Becker [29] used the Level 1 Green-Naghdi equations and Chang et al. [30] used a CFD model solving the RANS equations. These numerical approaches, and comparison to experiments therein, make clear that generation of higher harmonics, breaking waves and separation from the edges of the structure may all be important in certain regimes. These effects are not captured in the present model, and would all serve to reduce resonant (linear) amplitudes.

The higher order steady and low frequency oscillatory forces that the body experiences may also be important in the context of a moored wave energy device. For floating devices only the horizontal forces tend to be important; these may be modified for a submerged body as recognised by Longuet-Higgins [31] who investigated the mean horizontal second order drift force of submerged and floating bodies and the change in drift force due to wave breaking over a shallowly submerged horizontal cylinder. For a shallowly submerged taut-moored structure the vertical drift force may be of greater importance than the horizontal drift force, as the mean force from the waves needs to be taken into account in mooring design if the desired submergence is to be maintained. As has been shown above, the cylinder behaviour is strongly influenced by submergence, so any change in mean submergence is important. The mean second order vertical drift force may be calculated if the first order potential is known, and for a submerged oscillating circular cylinder, may be positive or negative Ogilvie [21]. Here we calculate the mean drift force for a fixed cylinder in waves 
and a moving submerged cylinder in still water, see figure 7 where we also show results from HydroStar calculated with the middle-field method [32]. See Appendix B for details. The behaviour of the diffracted and heave drift force is dominated by the first axisymmetric resonance. For the body moving in heave the drift force becomes negative (downwards) at approximately $k a=2.1$.

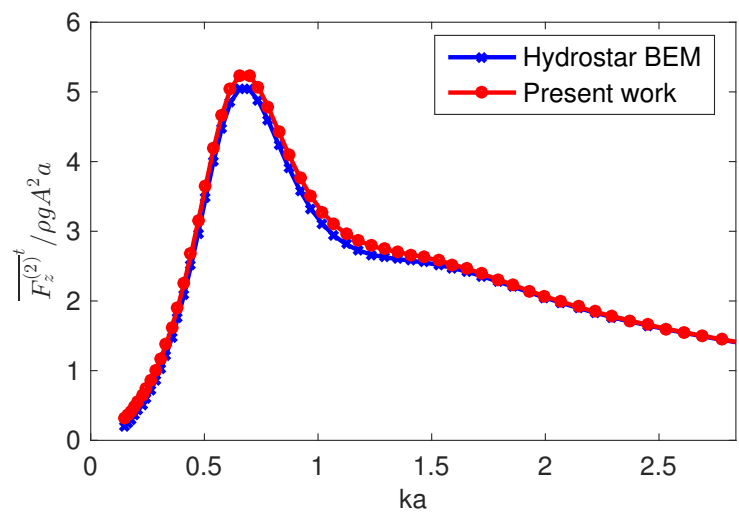

(a)

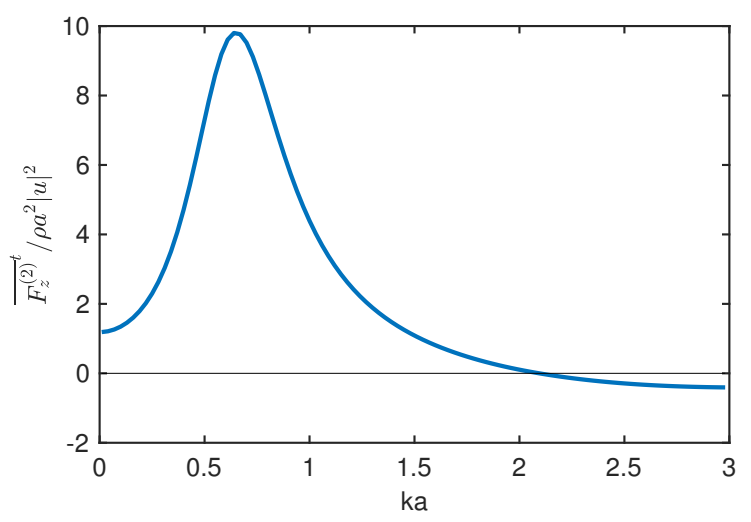

(b)

Figure 7: (a) Mean second order vertical drift force, diffraction only. (b) Radiation heave vertical drift. $h / a=5, T / a=0.3, s / h=0.06$.

\subsection{Four cylinder array}

One advantage of the semi-analytical model presented in this work over boundary element methods is the ability to rapidly assess arrays of devices. Here we will present some example results for a square arrangement of four cylinders. This arrangement is chosen because of its symmetry, which allows us to separate the contributions of different modes using symmetry theory (see e.g. Wolgamot et al. [33]) and because such a symmetric arrangement is known (at least in the surface piercing case - see, e.g. Evans and Porter [34]) to support near-trapped modes. Sloshing near-trapping effects for an array of four truncated cylinders were identified by Siddorn and Eatock Taylor [19] and radiation behaviour of the same array has been examined by Wolgamot et al. [35]. Near-trapping occurs when wave energy becomes trapped within an array or structure with little radiation to the far field but significant surface elevation within the array; depending on the coupling between fluid and body motion, there may be large effects on the hydrodynamic coefficients at adjacent frequencies. The array problem for submerged cylinders has a large number of relevant parameters including array spacing and arrangement, cylinder submergence, radius and thickness, and water depth; all of which influence the cylinder interactions. Here we have considered only a simple four cylinder array with fixed spacing.

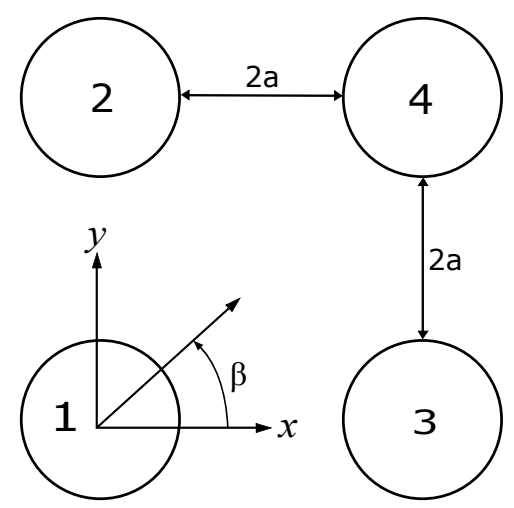

Figure 8: Cylinder array geometry 
The square array is defined by $h / a=5, T / a=0.3, s / h=0.06$ and spacing $4 a$ (centre to centre) which we will call array 1 , see figure 8 . We also present some results for the same array with shallower submergence $s / h=0.03$, which we will call array 2 . From equation (47) the effect of scattering by the array can be predicted by the properties of the matrix $X$. If $X$ is close to singular or ill conditioned there may be large amplification of the scattered waves associated with near-trapping or strong array interaction. Thus, as in Siddorn and Eatock Taylor [19], we can predict where near-trapping may occur by examining the condition number of the matrix $X$. Figure 9 shows the condition number of $X$ (using the 1-norm) plotted with frequency for both arrays. For submergence $s / h=0.06$ there is a clear peak in condition number at $k a=0.93$ indicating strong interaction and a small peak at $k a=1.65$, very close to the first near-trapping frequency which Siddorn and Eatock Taylor [19] identified at $k a=1.66$ for a surface piercing array with the same cylinder radii and layout. In contrast to the behaviour reported by Siddorn and Eatock Taylor, above $k a=2.3$ we do not see strong array interaction and near-trapping, as the submerged cylinders interact less with the high frequency waves which start to pass above the cylinders. At the shallower submergence of $s / h=0.03$ the peaks in condition number are shifted to lower frequency, as we might expect due to the resonances above an individual cylinder also being shifted to lower frequency. The condition numbers of the peaks are much larger in magnitude indicating strong interaction and there appears to be further interaction occurring in the range $k a=1-2$, but at higher frequency there is little variation in condition number.

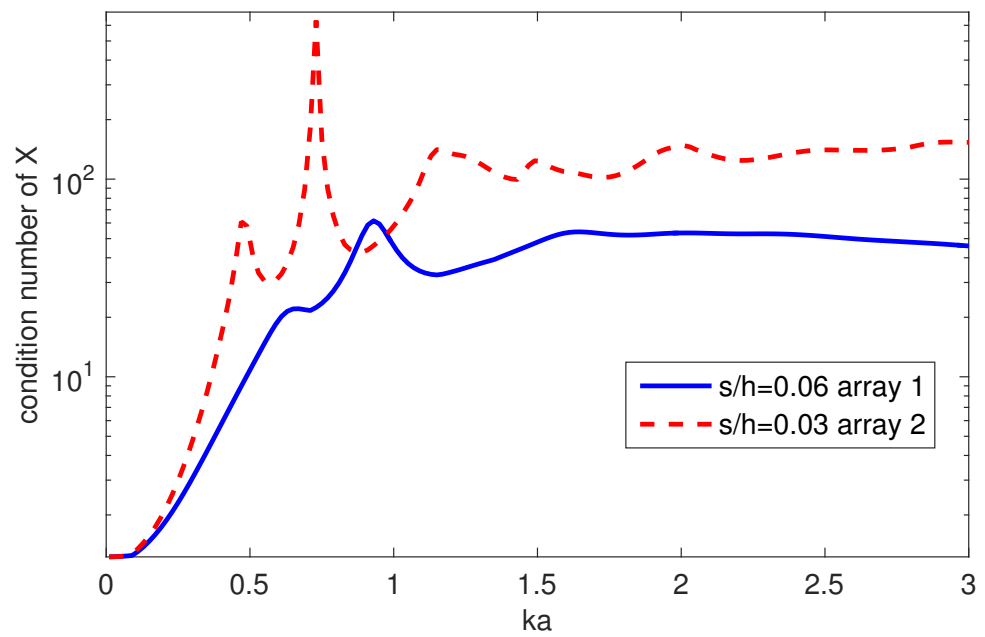

Figure 9: Condition number of the scattering matrix $X, T / a=0.3, h / a=5$.

Since the general $n \times n$ damping matrix $(B)$ is real and symmetric its eigenvalues $(\lambda)$ and eigenvectors $(\xi)$ are real. The eigenvectors form an orthonormal basis $(P)$ that can be used to diagonalise the damping matrix, with the eigenvector corresponding to a vector of body displacements and the eigenvalue proportional to the array damping. For an array of cylinders, the damping matrix may be modified as follows:

$$
P^{-1} B P=\operatorname{diag}\left(\lambda_{1}, \lambda_{2}, \ldots, \lambda_{n}\right)
$$

where the orthonormal basis is:

$$
P=\left(\xi_{1}, \xi_{2}, \ldots, \xi_{n}\right)
$$

Here we will only consider heave motion, such that $n$ is equal to the number of cylinders. This transformation is useful as it allows us to examine the contributions of array modes corresponding to different symmetries. Figure 10a shows the array damping in heave for array 1 and figure 10b shows the same array damping divided by that of a single cylinder. The rigid body motion mode where all cylinders oscillate in phase $\left(\xi_{1}=[1,1,1,1]\right)$ corresponds to the largest peak in array damping at $k a=0.94$ and the surface elevation at this frequency is shown in figure 12a. This feature is clearly associated with the peak in condition number at an almost identical frequency, and with the first resonance on top of the cylinder. However, the location of this peak is shifted away from the frequency of the first resonance for an isolated cylinder, emphasising the importance of array interactions. (This body mode also exhibits a 
minimum in damping at $k a=0.77$, as explained by Wolgamot et al. [35]).
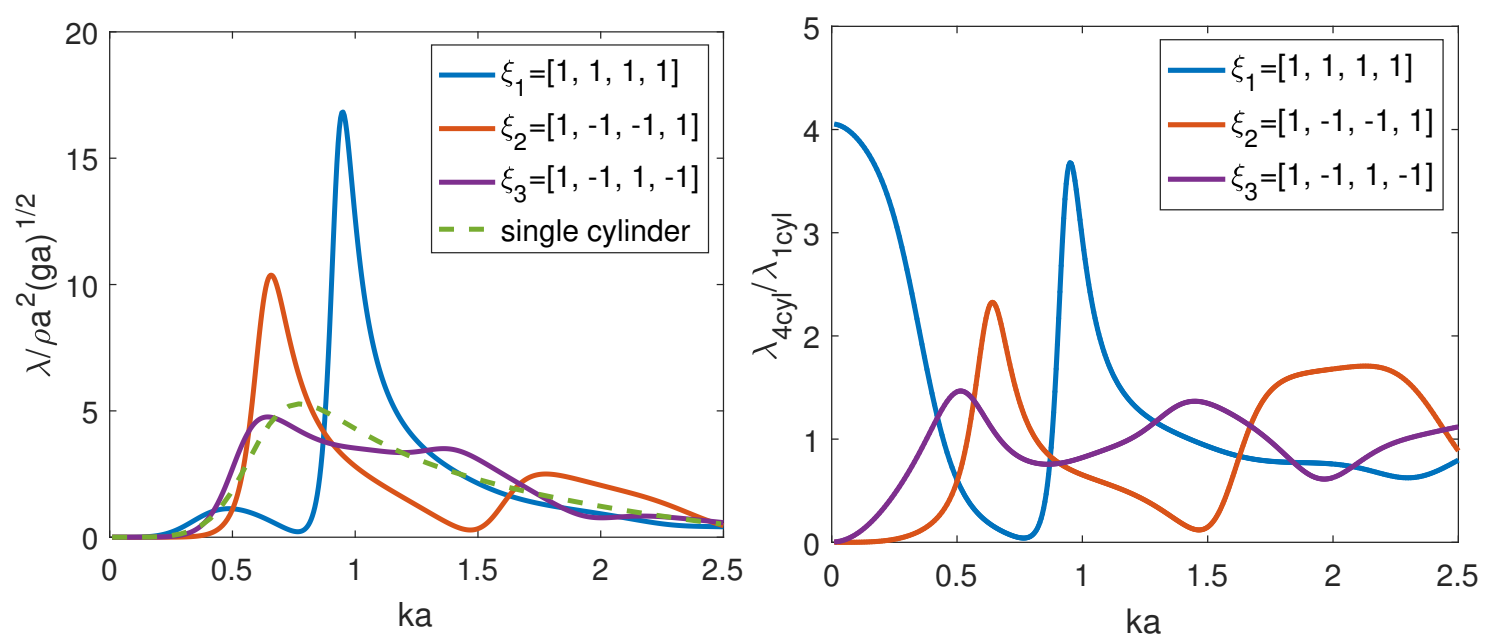

(a) Damping of heave body modes for a four cylinder array (b) Damping of heave body modes divided by single cylin(the body mode $[1,-1,1,-1]$ is of multiplicity 2 ). der damping.

Figure 10: Array 1 radiation damping.

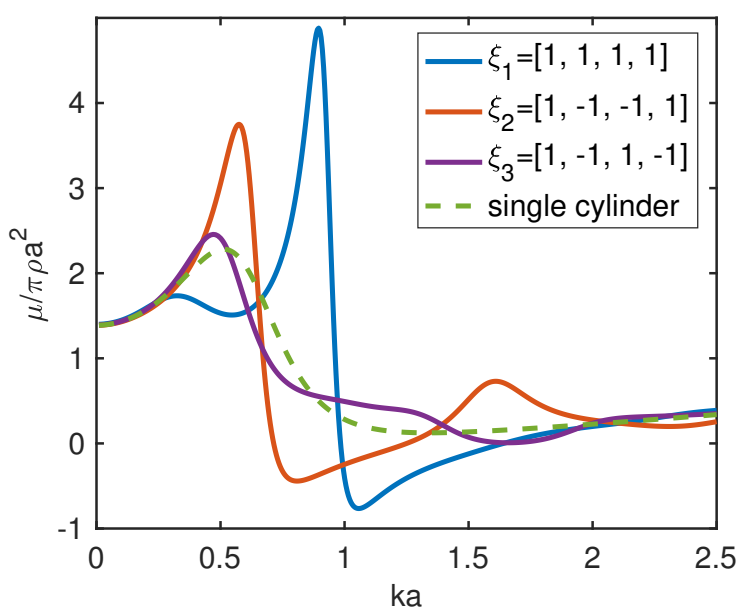

(a) Added mass of heave body modes for a four cylinder (b) array (the body mode $[1,-1,1,-1]$ is of multiplicity 2 ).

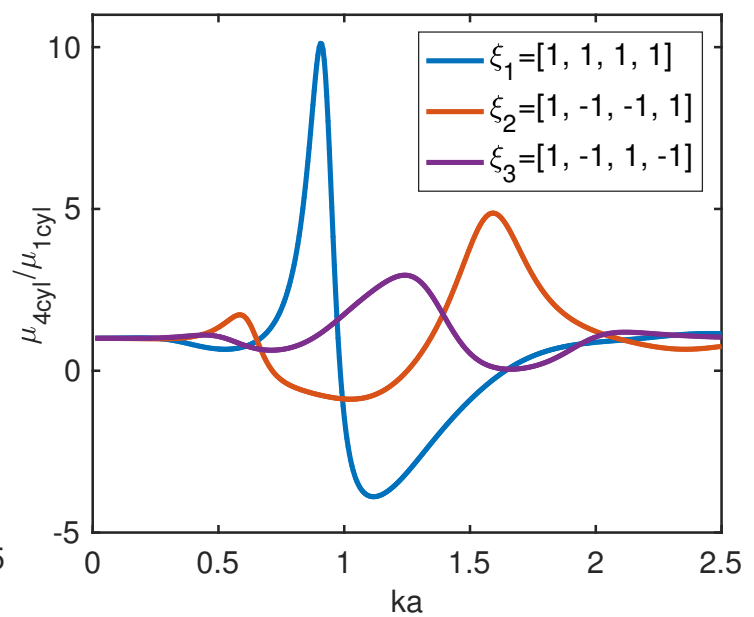

(b) Diagonal added mass of heave body modes divided by single cylinder added mass.

Figure 11: Array 1 added mass.

The added mass matrix is treated similarly (eigenvalues $\mu$ ), so that figure 11a shows the diagonalised added mass for each body mode and figure $11 \mathrm{~b}$ the same added mass divided by that of a single cylinder. We see large gradients in the added mass associated with the peaks in the damping as explained by, for example, McIver and Evans [4] for a single cylinder. There is a large increase in added mass over the single cylinder value at $k a=1.59$ for the $\xi_{2}=[1,-1,-1,1]$ mode. Figure $12 \mathrm{~b}$ shows the surface elevation for this mode and it can be seen that an asymmetric dipole-like resonance is excited over each cylinder, similar to the first resonance excited by surge of a single cylinder with peak surface elevation amplification at approximately $k a=1.92$. Array interactions hence allow the excitation of a dipole resonance that is not excited for a single cylinder oscillating only in heave. If the submergence is reduced to $s / h=0.03$ (array 2) we find the single cylinder dipole (surge) resonance peak at $k a=1.20$ and a peak in body mode 
$\xi_{2}=[1,-1,-1,1]$ added mass at $k a=1.25$. The surface elevation for this body mode is plotted in figure 13 and we see the wavefield above the cylinder strongly resembles a single cylinder dipole resonance. The free surface inside the array in figure 12a also strongly resembles the shape of the near trapped mode found for surface piercing cylinders (see e.g. Evans and Porter [34]). These examples show that the hydrodynamic properties of submerged cylinders in an array depend on the resonances above the cylinder, which are modified by interactions with other cylinders in the array.

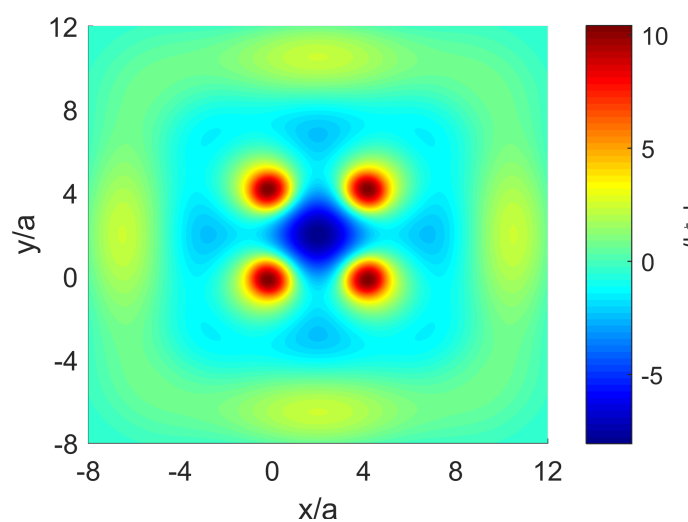

(a) Rigid body mode $\xi_{1}, k a=0.94, t \omega /(2 \pi)=0.196$.

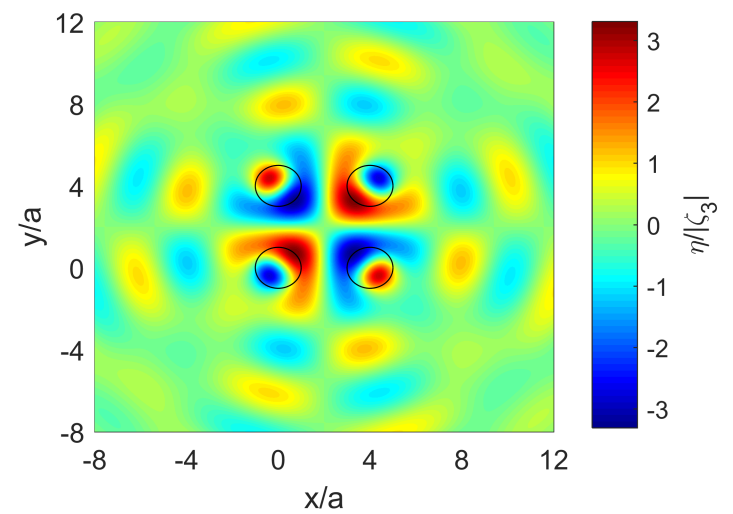

(b) Body mode $\xi_{2}=[1,-1,-1,1]$ in heave, $k a=1.59$, array 1 , the black circles indicate the cylinder positions, $t \omega /(2 \pi)=0.580$.

Figure 12: Surface elevation at maximum elevation above the cylinders, top view, radiation in heave, array 1 . Non-dimensionalised by the stroke of cylinder 1 in the original basis.

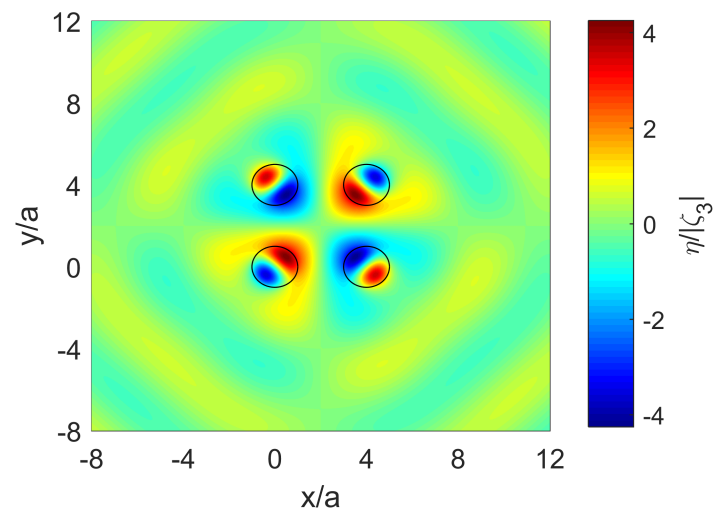

Figure 13: Surface elevation at maximum elevation above the cylinders, top view, radiation in heave, body mode $\xi_{2}=[1,-1,-1,1]$, array 2 , $k a=1.25, t \omega /(2 \pi)=0.623$.

Figure 14 shows the exciting force for each body mode (the dot product of the unit displacement vector with the vector of body exciting forces) for incident wave angles $\beta=0$ and 45 degrees. The damping in Figure 10a may be related to the heave exciting force by the Haskind relation, thus in Figure 14a a minimum in exciting force for the rigid body mode occurs at the same frequency as the minimum in damping. The rigid body mode is excited strongly for wave direction $\beta=0$ while the diagonal mode $\xi_{2}$ is significant for $\beta=45^{\circ}$. 


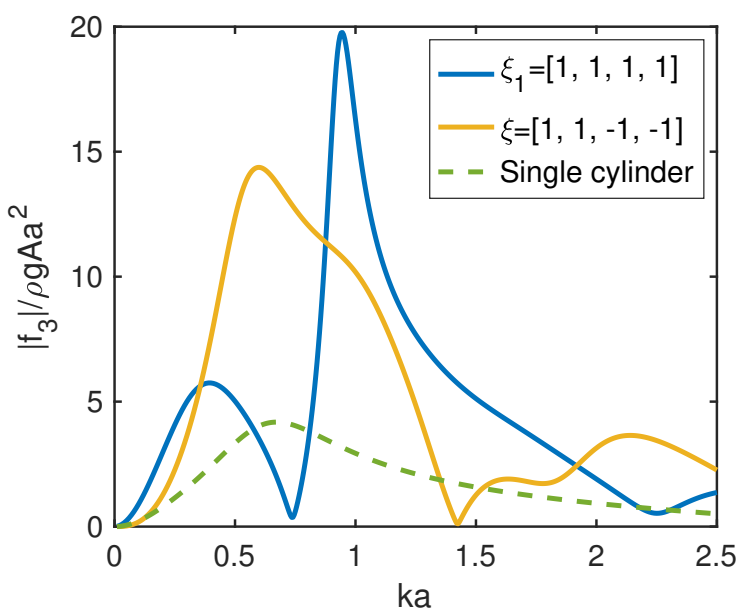

(a) $\beta=0$.

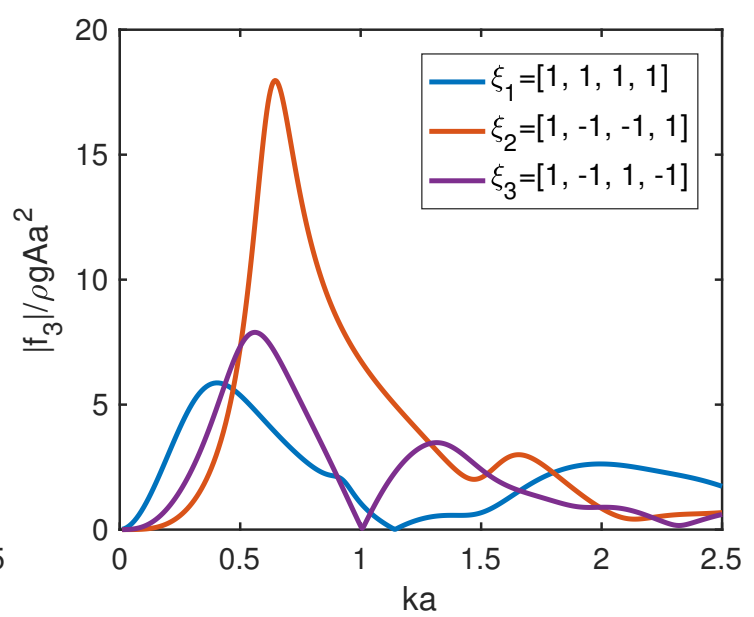

(b) $\beta=45^{\circ}$.

Figure 14: Heave exciting force for each body mode, array 1.

The example results shown in this section demonstrate the potential importance of array interactions for submerged cylinders.

\section{Conclusion}

The matched eigenfunction expansion and transform matrix methods have been applied to solve the linear diffraction and radiation problems for an arbitrary array of submerged cylinders and the method validated against commercial software. Resonances occurring in the fluid above a single cylinder driven by oscillations in heave and surge were characterised for different submergences. Large amplification of the surface elevation was seen at resonance for shallow submergences in both heave and surge motions, reducing applicability of the model for realistic sea states. A square array of four cylinders was studied and it was found that resonances above the cylinder remained important, but could be modified significantly by array interactions. The mean vertical drift force was calculated using direct pressure integration over the surface and may be relevant for submerged, taut-moored devices. There is much potential to use the model for future work to examine array interactions for larger arrays or different array configurations if the limitations of linear theory are kept in mind. It is apparent that an improved model for shallowly submerged cylinders is required to account for non-linearity in the fluid above the device if realistic sea states are to be considered for a wave energy device such as CETO.

\section{Acknowledgements}

The authors would like to thank Dr. P. Siddorn for sharing his work on arrays with us. GM would like to acknowledge funding from the Robert and Maude Gledden Scholarship. GM, HW and SD acknowledge the kind support of the Lloyds Register Foundation. The Lloyds Register Foundation supports the advancement of engineering related education, and funds research and development that enhances safety of life at sea, on land and in the air. HW also acknowledges financial support from Shell Australia. This work forms part of the activities conducted within the ARC Linkage Project 150100598.

\section{References}

[1] B. Borgarino, A. Babarit, P. Ferrant, Impact of wave interactions effects on energy absorption in large arrays of wave energy converters, Ocean Engineering 41 (2012) 79-88.

[2] Carnegie Clean Energy, Perth Wave Energy Project, URL http://carnegiewave.com/galleries/ perth-wave-energy-project/, accessed: 2017-06-15, 2015. 
[3] J. N. Newman, B. Sortland, T. Vinje, Added mass and damping of rectangular bodies close to the free surface, Journal of Ship Research 28 (4) (1984) 219-225.

[4] P. McIver, D. V. Evans, The occurrence of negative added mass in free-surface problems involving submerged oscillating bodies, Journal of Engineering Mathematics 18 (1) (1984) 7-22.

[5] P. A. Martin, L. Farina, Radiation of water waves by a heaving submerged horizontal disc, Journal of Fluid Mechanics 337 (1997) $365-379$.

[6] C. J. R. Garrett, Wave forces on a circular dock, Journal of Fluid Mechanics 46 (01) (1971) 129-139, ISSN 1469-7645.

[7] R. W. Yeung, Added mass and damping of a vertical cylinder in finite-depth waters, Applied Ocean Research 3 (3) (1981) 119-133, ISSN 0141-1187.

[8] S.-c. Jiang, Y. Gou, B. Teng, D.-z. Ning, Analytical solution of a wave diffraction problem on a submerged cylinder, Journal of Engineering Mechanics 140 (1) (2013) 225-232.

[9] S.-c. Jiang, Y. Gou, B. Teng, Water wave radiation problem by a submerged cylinder, Journal of Engineering Mechanics 140 (5) (2013) 06014003.

[10] H. Kagemoto, D. K. P. Yue, Interactions among multiple three-dimensional bodies in water waves: an exact algebraic method, Journal of Fluid Mechanics 166 (1986) 189-209, ISSN 1469-7645.

[11] B. H. Spring, P. L. Monkmeyer, Interaction of plane waves with vertical cylinders, in: Proceedings of the 14th international conference on coastal engineering, vol. 107, 1828-1845, 1974.

[12] M. J. Simon, Multiple scattering in arrays of axisymmetric wave-energy devices. Part 1. A matrix method using a plane-wave approximation, Journal of Fluid Mechanics 120 (1982) 1-25, ISSN 1469-7645.

[13] V. Twersky, Multiple scattering of radiation by an arbitrary configuration of parallel cylinders, The Journal of the Acoustical Society of America 24 (1) (1952) 42-46, ISSN 0001-4966.

[14] M. Ohkusu, Hydrodynamic forces on multiple cylinders in waves, in: Int. Symp. on the Dynamics of Marine Vehicles and Structures in Waves, 1974, 1974.

[15] C. M. Linton, D. V. Evans, The interaction of waves with arrays of vertical circular cylinders, Journal of Fluid Mechanics 215 (1990) 549-569, ISSN 1469-7645.

[16] M.-H. Kim, Interaction of waves with N vertical circular cylinders, Journal of Waterway, Port, Coastal, and Ocean Engineering 119 (6) (1993) 671-689, ISSN 0733-950X

[17] O. Yilmaz, A. Incecik, Analytical solutions of the diffraction problem of a group of truncated vertical cylinders, Ocean Engineering 25 (6) (1998) 385-394.

[18] O. Yilmaz, Hydrodynamic interactions of waves with group of truncated vertical cylinders, Journal of Waterway, Port, Coastal, and Ocean Engineering 124 (5) (1998) 272-279, ISSN 0733-950X.

[19] P. Siddorn, R. Eatock Taylor, Diffraction and independent radiation by an array of floating cylinders, Ocean Engineering 35 (13) (2008) 1289 - 1303, ISSN 0029-8018, doi:http://dx.doi.org/10.1016/j.oceaneng.2008.06.003.

[20] I. Chatjigeorgiou, V. Katsardi, Hydrodynamics and near trapping effects in arrays of multiple elliptical cylinders in waves, Ocean Engineering 157 (2018) 121 - 139, ISSN 0029-8018, doi:https://doi.org/10.1016/j.oceaneng.2018.03.045, URL http://www.sciencedirect.com/science/article/pii/S002980181830310X.

[21] T. F. Ogilvie, First-and second-order forces on a cylinder submerged under a free surface, Journal of Fluid Mechanics 16 (03) (1963) $451-472$.

[22] C. M. Lee, J. N. Newman, The vertical mean force and moment of submerged bodies under waves, Journal of Ship Research 15 (3).

[23] S. A. Mavrakos, The vertical drift force and pitch moment on axisymmetric bodies in regular waves, Applied Ocean Research 10 (4) (1988) 207-218.

[24] M. Abramowitz, I. A. Stegun, Handbook of Mathematical Functions, vol. 55, Courier Corporation, 1964.

[25] HydroStar user manual, Bureau Veritas, Paris, 2010.

[26] P. McIver, M. McIver, Trapped modes in the water-wave problem for a freely floating structure, Journal of Fluid Mechanics 558 (2006) 53-67.

[27] I. van Winsen, J. S. Bokhorst, R. H. M. Huijsmans, Calculation of Wave Height Dependent Force RAOs on Submerged Bodies in Close Proximity to the Free Surface, in: ASME 2013 32nd International Conference on Ocean, Offshore and Arctic Engineering, American Society of Mechanical Engineers, V001T01A017, 2013.

[28] J. Grue, Nonlinear water waves at a submerged obstacle or bottom topography, Journal of Fluid Mechanics 244 (1992) $455-476$.

[29] R. C. Ertekin, J. M. Becker, Nonlinear diffraction of waves by a submerged shelf in shallow water, Journal of Offshore Mechanics and Arctic Engineering 120 (4) (1998) 212-220.

[30] K.-A. Chang, T.-J. Hsu, P. L. F. Liu, Vortex generation and evolution in water waves propagating over a submerged rectangular obstacle: Part II: Cnoidal waves, Coastal Engineering 52 (3) (2005) 257-283.

[31] M. S. Longuet-Higgins, The mean forces exerted by waves on floating or submerged bodies with applications to sand bars and wave power machines, in: Proceedings of the Royal Society of London A: Mathematical, Physical and Engineering Sciences, vol. 352, The Royal Society, 463-480, 1977.

[32] X.-B. Chen, Middle-field formulation for the computation of wave-drift loads, Journal of Engineering Mathematics 59 (1) (2007) 61-82, ISSN 1573-2703, doi:10.1007/s10665-006-9074-x, URL https ://doi .org/10.1007/s10665-006-9074-x.

[33] H. A. Wolgamot, M. H. Meylan, C. D. Reid, Multiply heaving bodies in the time-domain: Symmetry and complex resonances, Journal of Fluids and Structures 69 (2017) 232-251.

[34] D. V. Evans, R. Porter, Trapping and near-trapping by arrays of cylinders in waves, Journal of Engineering Mathematics 35 (1-2) (1999) 149-179.

[35] H. A. Wolgamot, R. Eatock Taylor, P. H. Taylor, Radiation, trapping and near-trapping in arrays of floating truncated cylinders, Journal of Engineering Mathematics 91 (1) (2015) 17-35.

[36] T. F. Ogilvie, Second order hydrodynamic effects on ocean platforms, International Workshop on Ship and Platform Motions, 1983 (1983) 205-343.

[37] R. Zhao, O. M. Faltinsen, Interaction between current, waves and marine structures, in: International Conference on Numerical Ship Hydro- 
dynamics, 5th, 1989.

[38] H. Liang, O. M. Faltinsen, Y.-L. Shao, Application of a 2D harmonic polynomial cell (HPC) method to singular flows and lifting problems, Applied Ocean Research 53 (2015) 75-90

\section{Appendix A. Body Forces and Moments}

\section{Appendix A.1. Surge and Sway Force}

The complex amplitude of the surge force is found by integrating the fluid pressure over the vertical sides of the cylinder:

$$
\hat{F}_{1}=\left.\mathrm{i} \omega \rho \int_{c-h}^{-s} \int_{0}^{2 \pi} \phi\right|_{r=a} \cos (\theta) a \mathrm{~d} \theta \mathrm{d} z
$$

where the $\cos (\theta)$ factor follows from the $x$-direction component of the unit normal vector on the cylinder sides. Substituting the exterior region velocity potential (7):

$$
\frac{\hat{F}_{1}}{\omega \rho \pi a^{2}}=\frac{\mathrm{i}}{a} \sum_{m=0}^{\infty}\left(A_{1 m}+a_{1 m}+A_{-1 m}+a_{-1 m}\right) \int_{c-h}^{-s} Z_{m}(z) \mathrm{d} z .
$$

The sway force has a very similar derivation, but with $\cos (\theta)$ replaced by $\sin (\theta)$.

\section{Appendix A.2. Pitch and Roll Moment}

The contribution from the exterior potential to the pitch moment is give by:

$$
\begin{aligned}
\frac{\hat{F}_{5}^{E}}{\mathrm{i} \omega \rho} & =-\left.\int_{c-h}^{-s} \int_{0}^{2 \pi} \phi\right|_{r=a} \cos (\theta)\left(z_{0}-z\right) a \mathrm{~d} \theta \mathrm{d} z \\
& =\pi a^{2} \sum_{m=0}^{\infty}\left(A_{-1 m}+a_{-1 m}+A_{1 m}+a_{1 m}\right) \frac{1}{a} \int_{c-h}^{-s}\left(z-z_{0}\right) Z_{m}(z) \mathrm{d} z .
\end{aligned}
$$

The moment from the upper core homogeneous potential is:

$$
\begin{aligned}
\frac{\hat{F}_{5}^{U C H}}{\mathrm{i} \omega \rho} & =-\left.\int_{0}^{a} \int_{0}^{2 \pi} \phi\right|_{z=-s} r \cos (\theta) r \mathrm{~d} \theta \mathrm{d} r \\
& =-\pi a^{2}\left(N_{l}^{T}\right)^{-1 / 2}\left[\frac{\beta_{10}+\beta_{-10}}{\lambda_{0}} \frac{J_{2}\left(\lambda_{0} a\right)}{J_{1}\left(\lambda_{0} a\right)}+\sum_{l=1}^{\infty} \frac{\left(\beta_{1 l}+\beta_{-1 l}\right)}{\mathrm{i} \lambda_{l}} \frac{I_{2}\left(\mathrm{i} \lambda_{l} a\right)}{I_{1}\left(\mathrm{i} \lambda_{l} a\right)}\right] .
\end{aligned}
$$

The moment from the upper core inhomogeneous potential is:

$$
\begin{aligned}
\frac{\hat{F}_{5}^{U C I}}{\mathrm{i} \omega \rho} & =-\left.\int_{0}^{a} \int_{0}^{2 \pi} \phi\right|_{z=-s} r \cos (\theta) r \mathrm{~d} \theta \mathrm{d} r \\
& =\mathrm{i} u_{5} \frac{a^{2}}{4}\left(\frac{g}{\omega^{2}}-s\right) .
\end{aligned}
$$

When the lower core contributions are added, the total complex amplitude of the pitch moment is:

$$
\begin{aligned}
& \frac{\hat{F}_{5}}{\omega \rho \pi a^{2}}=\frac{\mathrm{i}}{a} \sum_{m=0}^{\infty}\left(A_{-1 m}\right.\left.+a_{-1 m}+A_{1 m}+a_{1 m}\right) \int_{c-h}^{-s}\left(z-z_{0}\right) Z_{m}(z) \mathrm{d} z \\
&+\mathrm{i}\left(a \frac{\alpha_{-10}+\alpha_{10}}{8}+c \sum_{n=1}^{\infty}\left(\alpha_{-1 n}+\alpha_{1 n}\right) \frac{I_{2}\left(\lambda_{n} a\right)}{I_{1}\left(\lambda_{n} a\right)} \frac{(-1)^{n}}{n \pi}\right)-\mathrm{i} u_{5} c\left(\frac{a^{2}}{8}-\frac{a^{4}}{48 c^{2}}\right) \\
&-\mathrm{i}\left(N_{l}^{T}\right)^{-1 / 2}\left[\frac{\beta_{10}-\beta_{-10}}{\lambda_{0}} \frac{J_{2}\left(\lambda_{0} a\right)}{J_{1}\left(\lambda_{0} a\right)}+\sum_{l=1}^{\infty} \frac{\left(\beta_{1 l}+\beta_{-1 l}\right)}{\mathrm{i} \lambda_{l}} \frac{I_{2}\left(\mathrm{i} \lambda_{l} a\right)}{I_{1}\left(\mathrm{i} \lambda_{l} a\right)}\right]+\mathrm{i} u_{5} \frac{a^{2}}{4}\left(\frac{g}{\omega^{2}}-s\right) .
\end{aligned}
$$


The roll moment is found in the same way as the pitch moment, replacing $\cos \theta$ by $\sin \theta$ in the integrals.

\section{Appendix B. Mean vertical drift force}

The mean (time averaged) second order drift force can be calculated from the first order potential only, with no need to calculate the second order potential. Here we calculate the mean vertical second order drift force on a submerged cylinder. Using perturbation theory we assume that all dependent variables can be expressed as a series in the small parameter $\epsilon$, for example:

$$
\Phi(r, \theta, z, t)=\epsilon \Phi^{(1)}(r, \theta, z, t)+\epsilon^{2} \Phi^{(2)}(r, \theta, z, t)+\ldots
$$

Following Ogilvie [36] the time average of the second order force for a fixed body is given by:

$$
{\overline{F^{(2)}}}^{t}=-\frac{1}{2} \rho \iint_{S} \mathbf{n}\left\{{\overline{\left(\frac{\partial \Phi^{(1)}}{\partial r}\right)^{2}}}^{t}+\frac{1}{r^{2}}{\overline{\left(\frac{\partial \Phi^{(1)}}{\partial \theta}\right)^{2}}}^{t}+{\overline{\left(\frac{\partial \Phi^{(1)}}{\partial z}\right)^{2}}}^{t}\right\} \mathrm{d} S,
$$

where overbar denotes the time average, $S$ is the wetted surface and $\mathbf{n}$ is a unit normal vector to the body surface directed into the body. This is equal to

$$
\begin{aligned}
{\overline{F^{(2)}}}^{t} & =-\frac{1}{4} \rho \iint_{S} \mathbf{n}\left\{\left|\frac{\partial \phi^{(1)}}{\partial r}\right|^{2}+\frac{1}{r^{2}}\left|\frac{\partial \phi^{(1)}}{\partial \theta}\right|^{2}+\left|\frac{\partial \phi^{(1)}}{\partial z}\right|^{2}\right\} \mathrm{d} S \\
& =-\frac{1}{4} \rho \iint_{S} \mathbf{n}|\vec{v}|_{S}^{2} \mathrm{~d} S .
\end{aligned}
$$

To obtain the vertical mean second order drift force for our cylinder we must integrate the modulus squared of the velocity over the top and bottom surfaces of the cylinder. Let us first consider a fixed body, where there is no contribution from the inhomogeneous potentials. In the lower core region the contribution to the velocity from the homogeneous potential is $\left(\boldsymbol{u}_{r}, \boldsymbol{u}_{\theta}\right.$ and $\boldsymbol{u}_{z}$ are unit vectors):

$$
\begin{aligned}
\hat{\vec{v}}^{L C H} & =\nabla \sum_{f=-\infty}^{\infty} \mathrm{e}^{\mathrm{i} f \theta}\left(\frac{\alpha_{f 0}}{2}\left(\frac{r}{a}\right)^{|f|}+\sum_{n=1}^{\infty} \alpha_{f n} \frac{I_{f}\left(\lambda_{n} r\right)}{I_{f}\left(\lambda_{n} a\right)} \cos \left(\lambda_{n}(z+h)\right)\right) \\
& =\boldsymbol{u}_{r} \sum_{f=-\infty}^{\infty} \mathrm{e}^{\mathrm{i} f \theta}\left(\frac{|f| \alpha_{f 0}}{2 r}\left(\frac{r}{a}\right)^{|f|}+\sum_{n=1}^{\infty} \alpha_{f n} \frac{I_{f}^{\prime}\left(\lambda_{n} r\right)}{I_{f}\left(\lambda_{n} a\right)} \cos \left(\lambda_{n}(z+h)\right)\right) \\
& +\boldsymbol{u}_{\theta} \frac{1}{r} \sum_{f=-\infty}^{\infty} \mathrm{i} f \mathrm{e}^{\mathrm{i} f \theta}\left(\frac{\alpha_{f 0}}{2}\left(\frac{r}{a}\right)^{|f|}+\sum_{n=1}^{\infty} \alpha_{f n} \frac{I_{f}\left(\lambda_{n} r\right)}{I_{f}\left(\lambda_{n} a\right)} \cos \left(\lambda_{n}(z+h)\right)\right) \\
& -\boldsymbol{u}_{z}\left(\sum_{f=-\infty}^{\infty} \mathrm{e}^{\mathrm{i} f \theta} \sum_{n=1}^{\infty} \alpha_{f n} \lambda_{n} \frac{I_{f}\left(\lambda_{n} r\right)}{I_{f}\left(\lambda_{n} a\right)} \sin \left(\lambda_{n}(z+h)\right)\right.
\end{aligned}
$$


Similarly from the upper core homogeneous potential:

$$
\begin{aligned}
\hat{\vec{v}}^{U C H} & =\nabla \sum_{f=-\infty}^{\infty} \mathrm{e}^{\mathrm{i} f \theta}\left(\beta_{f 0} \frac{J_{f}\left(\lambda_{0} r\right)}{J_{f}\left(\lambda_{0} a\right)} U_{0}(z)+\sum_{l=1}^{\infty} \beta_{f l} \frac{I_{f}\left(\mathrm{i} \lambda_{l} r\right)}{I_{f}\left(\mathrm{i} \lambda_{l} a\right)} U_{l}(z)\right) \\
& =\boldsymbol{u}_{r} \sum_{f=-\infty}^{\infty} \mathrm{e}^{\mathrm{i} f \theta}\left(\beta_{f 0} \frac{J_{f}^{\prime}\left(\lambda_{0} r\right)}{J_{f}\left(\lambda_{0} a\right)} U_{0}(z)+\sum_{l=1}^{\infty} \beta_{f l} \frac{I_{f}^{\prime}\left(\mathrm{i} \lambda_{l} r\right)}{I_{f}\left(\mathrm{i} \lambda_{l} a\right)} U_{l}(z)\right) \\
& +\boldsymbol{u}_{\theta} \frac{1}{r} \sum_{f=-\infty}^{\infty} \mathrm{i} f \mathrm{e}^{\mathrm{i} f \theta}\left(\beta_{f 0} \frac{J_{f}\left(\lambda_{0} r\right)}{J_{f}\left(\lambda_{0} a\right)} U_{0}(z)+\sum_{l=1}^{\infty} \beta_{f l} \frac{I_{f}\left(\mathrm{i} \lambda_{l} r\right)}{I_{f}\left(\mathrm{i} \lambda_{l} a\right)} U_{l}(z)\right) \\
& +\boldsymbol{u}_{z} \sum_{f=-\infty}^{\infty} \mathrm{e}^{\mathrm{i} f \theta}\left(\beta_{f 0} \frac{J_{f}\left(\lambda_{0} r\right)}{J_{f}\left(\lambda_{0} a\right)} \frac{\partial U_{0}(z)}{\partial z}+\sum_{l=1}^{\infty} \beta_{f l} \frac{I_{f}\left(\mathrm{i} \lambda_{l} r\right)}{I_{f}\left(\mathrm{i} \lambda_{l} a\right)} \frac{\partial U_{l}(z)}{\partial z}\right) .
\end{aligned}
$$

Then the mean vertical drift force is:

$$
{\overline{F_{z}^{(2)}}}^{t}=\frac{1}{4} \rho \int_{0}^{2 \pi} \int_{0}^{a} \mathbf{u}_{z}\left\{\left|\vec{v}^{U C}\right|_{z=-s}^{2}-\left|\vec{v}^{L C}\right|_{z=c-h}^{2}\right\} r \mathrm{~d} r \mathrm{~d} \theta .
$$

Now considering the radiation problem for a heaving submerged cylinder, following Ogilvie [36], equation (73) and ignoring rotations, the second order force can be written as:

$$
F^{(2)}=-\rho \iint_{S} \mathbf{n}\left\{\frac{\partial \Phi^{(2)}}{\partial t}+\frac{1}{2}\left|\nabla \Phi^{(1)}\right|^{2}+\vec{Z}^{(1)} \cdot \nabla \frac{\partial \Phi^{(1)}}{\partial t}\right\} d S,
$$

where $Z^{(1)}=\left(Z_{1}^{(1)}, Z_{2}^{(1)}, Z_{3}^{(1)}\right)$ is the vector of body translations to first order and $S$ is the equilibrium wetted surface of the body. The time averaged second order force for a cylinder moving in heave is then given by integrals over the top and bottom surfaces $\left(S_{z}\right)$ :

$$
{\overline{F_{z}^{(2)}}}^{t}=-\frac{1}{2} \rho \iint_{S_{z}} \mathbf{n}\left\{{\overline{\left(\frac{\partial \Phi^{(1)}}{\partial r}\right)^{2}}}^{t}+\frac{1}{r^{2}}{\overline{\left(\frac{\partial \Phi^{(1)}}{\partial \theta}\right)^{2}}}^{t}+{\overline{\left(\frac{\partial \Phi^{(1)}}{\partial z}\right)^{2}}}^{t}+2{\overline{2 Z_{3}^{(1)}} \frac{\partial}{\partial z} \frac{\partial \Phi^{(1)}}{\partial t}}^{t}\right\} \mathrm{d} S_{z}
$$

Where, assuming the body motion is harmonic, $Z_{3}^{(1)}(t)=\operatorname{Re}\left\{\zeta_{3} e^{i \omega t}\right\}$. We must now include the inhomogeneous components of the potential, for which the derivatives are simple to evaluate. In the last term in equation (B.8) we have $z$ derivative components. The homogeneous components for this radiation correction term are zero when evaluated on the body surface and the inhomogeneous components from the upper and lower cores cancel so that the only terms which contribute are those also present for the fixed cylinder, albeit with different values of the potential.

Evaluation of the radial integrals of the top and bottom homogeneous velocity components presented some challenges due to the presence of multiple non-standard Bessel function product integrals; as such the vertical drift force was calculated by numerical integration. The velocity was calculated on the top and bottom surfaces of the cylinder for a range of radial points and then integrated over the domain. The angular integral can be evaluated analytically for a finite number of Fourier modes. A convergence study was conducted to determine the number of radial points as well as Fourier, exterior evanescent and internal modes required to achieve a converged solution. Evaluation of the mean drift force by direct pressure integration is known to cause some difficulties at the cylinder edge (e.g. Zhao and Faltinsen [37], Liang et al. [38]) as the velocity potential has a singularity, which is poorly captured by the eigenfunctions used. Up to two hundred exterior evanescent modes were used to achieve adequate convergence. 